\title{
Hilbert's 14th problem over finite fields and a conjecture on the cone of curves
}

\author{
Burt Totaro
}

\begin{abstract}
We give the first examples over finite fields of rings of invariants that are not finitely generated. (The examples work over arbitrary fields, for example the rational numbers.) The group involved can be as small as three copies of the additive group. The failure of finite generation comes from certain elliptic fibrations or abelian surface fibrations having positive Mordell-Weil rank. Our work suggests a generalization of the MorrisonKawamata cone conjecture on Calabi-Yau fiber spaces to klt Calabi-Yau pairs. We prove the conjecture in dimension two under the assumption that the anticanonical bundle is semi-ample.
\end{abstract}

\section{Introduction}

Hilbert's 14th problem asks whether the ring of invariants of any representation of a linear algebraic group is finitely generated over the base field. Nagata gave the first counterexample, using a representation of $\left(G_{\mathrm{a}}\right)^{13}$, where $G_{\mathrm{a}}$ denotes the additive group [Nag58]. In his example, the representation is defined over a field of large transcendence degree over the prime field (of any characteristic). Mukai simplified Nagata's construction, showing that there are representations of $\left(G_{\mathrm{a}}\right)^{3}$ over the complex numbers whose ring of invariants is not finitely generated [Muk01].

Mukai relates the problem of finite generation of rings of invariants to a natural question in algebraic geometry: when is the total coordinate ring of a projective variety finitely generated? The deepest known result is the Birkar-Cascini-Hacon-McKernan theorem that the total coordinate ring of a Fano variety in characteristic zero is finitely generated [BCHM06, Corollary 1.3.1]. Nagata and Mukai give counterexamples to finite generation for certain varieties (blow-ups of projective space) just outside the realm of Fano varieties. The precise border between finite and infinite generation remains to be understood.

Mukai's construction yields representations whose coefficients are 'general' complex numbers. In this paper, we show that Mukai's three best examples can all be realized over finite fields and over the rational numbers, in fact with simple explicit coefficients. We give examples over all fields, including the field of order two. These are the first published counterexamples to Hilbert's 14th problem over finite fields. (In retrospect, some examples over finite fields can be constructed using the work of Manin [Man64], Tate [Tat65], or Shioda [Shi72] on elliptic surfaces.) For some classes of examples, we characterize exactly when finite generation holds and when it does not.

Theorem 0.1. Let $k$ be any field. Then there are linear representations over $k$ of $\left(G_{\mathrm{a}}\right)^{3}$ on $A^{18}$, of $\left(G_{\mathrm{a}}\right)^{4}$ on $A^{16}$, and of $\left(G_{\mathrm{a}}\right)^{6}$ on $A^{18}$ whose rings of invariants are not finitely generated. The representations are defined explicitly.

Received 25 September 2007, accepted in final form 19 February 2008.

2000 Mathematics Subject Classification 13A50, 14E30, 14J32.

Keywords: finite generation, ring of invariants, elliptic fibration, Calabi-Yau fiber space, klt pair, cone conjecture.

This journal is (c) Foundation Compositio Mathematica 2008. 


\section{HILBERT'S 14TH PROBLEM}

Here is the geometric idea. The basic ingredient of Nagata's examples (especially as simplified by Steinberg [Ste97]) is the existence of non-torsion line bundles of degree zero on an elliptic curve. There are no such line bundles on curves over finite fields, but the generic fiber of an elliptic fibration over a finite field can have infinite Mordell-Weil group, and that turns out to be enough. Precisely, we encounter known elliptic fibrations of blow-ups of $\mathbf{P}^{2}$ and $\mathbf{P}^{3}$, and what seems to be a new fibration of a blow-up of $\mathbf{P}^{5}$ by abelian surfaces $(\S 6)$. It would be interesting to describe the geometry of these rational abelian fibrations of $\mathbf{P}^{5}$ in more detail. For example, are these fibrations completely integrable systems with respect to some Poisson structure?

Our main results (Theorems 5.2 and 7.2) relate finite generation of the total coordinate ring, in some situations, to finiteness of a certain Mordell-Weil group. We conclude with a more general conjecture, saying that the cone of curves of any variety with semi-ample anticanonical bundle is controlled by a group, which may be infinite. This would follow from a generalization of the conjectures of Kawamata and Morrison on Calabi-Yau fiber spaces to allow klt pairs (Conjecture 8.1). We prove the conjecture for smooth projective surfaces with semi-ample anticanonical bundle, the new case being that of rational elliptic surfaces (Theorem 8.2).

\section{Another example}

The smallest known representation of an algebraic group for which finite generation fails is Freudenburg's 11-dimensional representation of a unipotent group $\left(G_{\mathrm{a}}\right)^{4} \rtimes G_{\mathrm{a}}$ over the rational numbers [Fre07], based on an example by Kuroda [Kur04]. It would be interesting to know whether there are such low-dimensional examples over finite fields.

There are broader forms of Hilbert's 14th problem, for example about actions of algebraic groups on arbitrary affine varieties. Since even the most specific form of the problem, regarding linear representations, has a negative answer, we focus on that case.

\section{Mukai's method}

In this section, we summarize Mukai's geometric approach to producing counterexamples to Hilbert's 14th problem.

We use the following result by Mukai [Muk01]. (He works over the complex numbers, but his proof is elementary and works over any field.)

Theorem 2.1. For any $n \geqslant r \geqslant 3$, let $X$ be the blow-up of projective space $\mathbf{P}^{r-1}$ at $n$ distinct rational points $p_{1}, \ldots, p_{n}$, not contained in a hyperplane, over a field $k$. Let $G \cong\left(G_{\mathrm{a}}\right)^{n-r}$ be the subgroup of $\left(G_{\mathrm{a}}\right)^{n}$ which is the kernel of a linear map $A^{n} \rightarrow A^{r}$ corresponding to the points $p_{i}$. Let $\left(G_{\mathrm{a}}\right)^{n}$ act on $V=A^{2 n}$ by

$$
\left(t_{1}, \ldots, t_{n}\right)\left(x_{1}, \ldots, x_{n}, y_{1}, \ldots, y_{n}\right)=\left(x_{1}, \ldots, x_{n}, y_{1}+t_{1} x_{1}, \ldots, y_{n}+t_{n} x_{n}\right) .
$$

Then the ring of invariants $O(V)^{G}$ is isomorphic to the total coordinate ring of $X$,

$$
T C(X):=\bigoplus_{a, b_{1}, \ldots, b_{n} \in \mathbf{Z}} H^{0}\left(X, a H-b_{1} E_{1}-\cdots-b_{n} E_{n}\right) \cong \bigoplus_{L \in \operatorname{Pic} X} H^{0}(X, L),
$$

where $H$ is the pullback of the hyperplane line bundle on $\mathbf{P}^{r-1}$ and $E_{1}, \ldots, E_{n}$ are the exceptional divisors in $X$.

Therefore, to give examples of rings of invariants which are not finitely generated, it suffices to exhibit arrangements of points on projective space such that the total coordinate ring of the blow-up $X$ is not finitely generated. 


\section{B. TOTARO}

Define a pseudo-isomorphism between smooth projective varieties to be a birational map which is an isomorphism outside subsets of codimension at least two. This notion can be useful for singular varieties, but our applications only involve smooth varieties. (For example, a pseudo-isomorphism between smooth projective surfaces is an isomorphism.) A pseudo-isomorphism $X \rightarrow Y$ induces an isomorphism $\operatorname{Pic}(X) \rightarrow \operatorname{Pic}(Y)$ by taking the proper transform of divisors. Define a $(-1)$ divisor $D$ on a projective variety $X$ to be the proper transform under some pseudo-isomorphism $X \rightarrow X^{\prime}$ of the exceptional divisor for a morphism $X^{\prime} \rightarrow Y$ which is the blow-up of a smooth point. (For example, a (-1)-curve on a surface is simply a curve isomorphic to $\mathbf{P}^{1}$ with self-intersection -1 , by Castelnuovo.) Mukai observes that every (-1)-divisor is indecomposable in the monoid of effective line bundles on $X$ (line bundles $L$ with $H^{0}(X, L) \neq 0$ ). Also, two different (-1)-divisors represent different elements of $\operatorname{Pic}(X)$. Therefore, if a variety $X$ contains infinitely many $(-1)$-divisors, then the monoid of effective line bundles on $X$ is not finitely generated, and so the total coordinate ring of $X$ is not finitely generated [Muk01, Lemma 3].

Lemma 2.2 gives a way to ensure that a blow-up $X$ of projective space contains infinitely many $(-1)$-divisors. To state it, define a birational map $\Psi: \mathbf{P}^{r-1} \rightarrow \mathbf{P}^{r-1}$ by

$$
\left[x_{1}, \ldots, x_{r}\right] \mapsto\left[\frac{1}{x_{1}}, \ldots, \frac{1}{x_{r}}\right] .
$$

It contracts the $r$ coordinate hyperplanes to the $r$ coordinate points. A birational map between two projective spaces which is projectively equivalent to $\Psi$ is called a standard Cremona transformation. We note that $\Psi$ lifts to a pseudo-isomorphism from the blow-up of $\mathbf{P}^{r-1}$ at the $r$ coordinate points to itself. In modern terms, this pseudo-isomorphism can be described as a composite of several flops.

We say that an arrangement of $n$ points in $\mathbf{P}^{r-1}, n \geqslant r \geqslant 3$, is in linear general position if no $r$ of the points are contained in a hyperplane. (In particular, the points are all distinct.) Given $n$ points in linear general position, we can perform the standard Cremona transformation on any $r$ of the $n$ points; this gives a different arrangement of $n$ points in a projective space. They need not be in linear general position. We say that an arrangement of $n$ points in $\mathbf{P}^{r-1}$ is in Cremona general position if they are in linear general position and this remains true after any finite sequence of standard Cremona transformations on $r$-tuples of the points.

The key point of Mukai's method is the following result.

Lemma 2.2. Let $p_{1}, \ldots, p_{n}, n \geqslant r \geqslant 3$, be points in projective space $\mathbf{P}^{r-1}$ over a field $k$ which are in Cremona general position. If

$$
\frac{1}{2}+\frac{1}{r}+\frac{1}{n-r} \leqslant 1
$$

then the blow-up $X$ of $\mathbf{P}^{r-1}$ at $p_{1}, \ldots, p_{n}$ contains infinitely many $(-1)$-divisors. Therefore, the total coordinate ring of $X$ is infinitely generated, and the corresponding representation of $\left(G_{\mathrm{a}}\right)^{n-r}$ of dimension $2 n$ over $k$ has infinitely generated ring of invariants.

For clarity, we recall the proof of Lemma 2.2. Clearly $X$ contains $n(-1)$-divisors over the $n$ points $p_{1}, \ldots, p_{n}$. However, since $p_{1}, \ldots, p_{n}$ are in Cremona general position, we can perform Cremona transformations on $r$-tuples of points in $p_{1}, \ldots, p_{n}$ any number of times. This shows that $X$ is pseudo-isomorphic to a blow-up of $\mathbf{P}^{r-1}$ in many other ways, and so we find other $(-1)$-divisors on $X$. The inequality on $n$ ensures, by a purely combinatorial argument, that the resulting $(-1)$ divisors on $X$ have arbitrarily large degrees (when projected down to divisors on $\mathbf{P}^{r-1}$ ), and so there are infinitely many of them. To describe this argument in more detail, define a symmetric bilinear form on $\operatorname{Pic}(X)$ by $H^{2}=r-2, E_{i}^{2}=-1, H \cdot E_{j}=0$, and $E_{i} \cdot E_{j}=0$ for $i \neq j$. We have a canonical identification of $\operatorname{Pic}(X)$ with $\mathbf{Z}^{n+1}=\mathbf{Z} H \oplus \mathbf{Z} E_{1} \oplus \cdots \oplus \mathbf{Z} E_{n}$ for every blow-up $X$ of an $(r-1)$-dimensional projective space at an ordered set of $n$ points. So the standard Cremona 


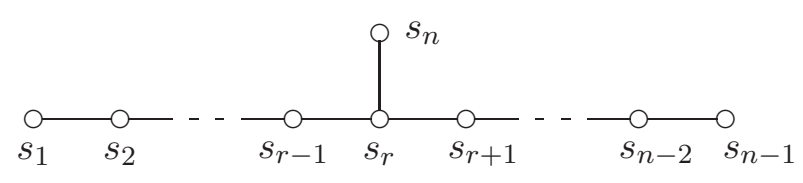

Figure 1. $T_{2, r, n-r}$ Dynkin diagram.

transformation at the points $p_{1}, \ldots, p_{r}$ determines a well-defined automorphism of $\mathbf{Z}^{n+1}$, which we compute to be the reflection $s_{n}$ orthogonal to $H-E_{1}-\cdots-E_{r}$. Switching points $p_{i}$ and $p_{i+1}$ acts on $\mathbf{Z}^{n+1}$ by the reflection $s_{i}$ orthogonal to $E_{i}-E_{i+1}$ for $1 \leqslant i \leqslant n-1$. We compute (it is also clear geometrically) that the Cremona action on $\operatorname{Pic}(X)=\mathbf{Z}^{n+1}$ fixes the anticanonical class of $X$, $-K_{X}=r H-(r-2) E_{1}-\cdots-(r-2) E_{n}$. Together the reflections $s_{1}, \ldots, s_{n-1}, s_{n}$ generate the Weyl group with Dynkin diagram $T_{2, r, n-r}$ (see Figure 1), and the inequality in Lemma 2.2 is just what is needed to ensure that this Weyl group is infinite.

It is clear that very general $n$-tuples of points in projective space (that is, $n$-tuples outside of countably many proper subvarieties of $\left.\left(\mathbf{P}^{r-1}\right)^{n}\right)$ are in Cremona general position. Thus, Lemma 2.2 gives counterexamples to Hilbert's 14th problem over the complex numbers (or any uncountable field). However, this argument leaves it unclear whether there are any arrangements in Cremona general position over the rational numbers or over finite fields. In the next section we construct such arrangements. These occur in the most interesting cases of Lemma 2.2 , where $1 / 2+1 / r+1 /(n-r)=$ 1 , corresponding to the affine Weyl groups of type $E_{8}^{(1)}(n=9, r=3$ or 6$)$ or $E_{7}^{(1)}(n=8, r=4)$.

As a historical note, in 1929 Coble found that the blow-up of $\mathbf{P}^{2}$ at nine very general points contained infinitely many $(-1)$-curves $[\mathrm{Cob} 29, \S 9]$, but the first proof to modern standards was given by Nagata in 1960 [Nag60, Lemma 2.5 and Theorem 4a].

\section{The method, with elementary proofs}

In this section we prove Theorem 0.1 over any sufficiently large finite field and over any infinite field. This turns out to require only some simple geometry, following the ideas of Coble and Dolgachev.

By Lemma 2.2, Theorem 0.1 will follow if we can produce explicit examples of 9-tuples of points in $\mathbf{P}^{2}$ in Cremona general position, or 8 -tuples in $\mathbf{P}^{3}$, or 9 -tuples in $\mathbf{P}^{5}$. The following lemmas do this, over sufficiently large finite fields and over infinite fields. The idea is to use arrangements of points which are special in one way (for example, we take nine points in $\mathbf{P}^{2}$ which are the intersection of two cubics), but not too special.

In what follows, a cubic in $\mathbf{P}^{2}$ denotes an effective divisor of degree three; it is not assumed to be irreducible (likewise for conics, quadrics in higher-dimensional projective spaces, and so on). We say that a cubic is irreducible if it is irreducible and reduced (that is, the corresponding cubic form is irreducible). A pencil of cubics in $\mathbf{P}^{2}$ means a linear system $\mathbf{P}^{1}$ of cubics; equivalently, it is a two-dimensional linear subspace of the vector space of cubic forms in three variables. We say that two cubics in $\mathbf{P}^{2}$ over a field $k$ intersect in nine given points if those are all of the intersection points over the algebraic closure of $k$.

Lemma 3.1. Let $p_{1}, \ldots, p_{9}$ be nine distinct rational points in $\mathbf{P}^{2}$ over a field $k$. Suppose that there are cubics $C_{1}$ and $C_{2}$ whose intersection is the set $\left\{p_{1}, \ldots, p_{9}\right\}$. Then no three of $p_{1}, \ldots, p_{9}$ lie on a line if and only if all cubics in the pencil spanned by $C_{1}$ and $C_{2}$ are irreducible. 


\section{B. TOTARO}

Proof. Suppose that a cubic $C_{1}$ in the pencil is reducible. Then we can write $C_{1}=L+D$ for some line $L$ and conic $D$. For any other cubic $C_{2}$ in the pencil,

$$
\left\{p_{1}, \ldots, p_{9}\right\}=C_{1} \cap C_{2}=\left(L \cap C_{2}\right) \cup\left(D \cap C_{2}\right) .
$$

Since $C_{2}$ intersects $C_{1}$ transversely, three of these points are on the line $L$. Conversely, suppose that $p_{1}, p_{2}, p_{3}$ lie on a line $L$. There is a unique cubic on $L$ through $p_{1}, p_{2}, p_{3}$. Since we have a pencil (a $\mathbf{P}^{1}$ ) of cubics in $\mathbf{P}^{2}$ through $p_{1}, p_{2}, p_{3}$, at least one cubic $C_{1}$ in the pencil must contain $L$. Thus, $C_{1}$ is reducible.

Corollary 3.2. Let $p_{1}, \ldots, p_{9}$ be nine distinct rational points in $\mathbf{P}^{2}$ over a field $k$ which are the intersection of two cubics. Suppose that no three of $p_{1}, \ldots, p_{9}$ lie on a line. Then $p_{1}, \ldots, p_{9}$ are in Cremona general position. Therefore the blow-up $X$ of $\mathbf{P}^{2}$ at $p_{1}, \ldots, p_{9}$ has infinitely generated total coordinate ring, and the corresponding 18-dimensional representation of $\left(G_{\mathrm{a}}\right)^{6}$ over $k$ has infinitely generated ring of invariants.

The first part of Corollary 3.2 was proved first by Manin [Man64, Lemma 3].

Proof. The Cremona action on $\operatorname{Pic}(X) \cong \mathbf{Z}^{10}$ fixes the anticanonical class $-K_{X}=3 H-E_{1}-$ $\cdots-E_{9}$. Therefore, performing a standard Cremona transformation on any three of $p_{1}, \ldots, p_{9}$ transforms a cubic through $p_{1}, \ldots, p_{9}$ into a cubic through the new points $p_{1}^{\prime}, \ldots, p_{9}^{\prime}$. Since $p_{1}, \ldots, p_{9}$ are the intersection of two cubics in $\mathbf{P}^{2}$, this remains true after performing a standard Cremona transformation on any three of $p_{1}, \ldots, p_{9}$. Since no three of $p_{1}, \ldots, p_{9}$ lie on a line, all cubics in the pencil of cubics through these points are irreducible by Lemma 3.1. The point is that this remains true of the proper transforms of these cubics on the 'new' $\mathbf{P}^{2}$. Therefore, no three of $p_{1}^{\prime}, \ldots, p_{9}^{\prime}$ lie on a line. Thus, $p_{1}^{\prime}, \ldots, p_{9}^{\prime}$ satisfy the same properties we assumed for $p_{1}, \ldots, p_{9}$. We can repeat this process any number of times. So $p_{1}, \ldots, p_{9}$ are in Cremona general position. Lemma 2.2 gives the rest.

Corollary 3.2 gives counterexamples to Hilbert's 14th problem over all sufficiently large finite fields, and over all infinite fields; we give explicit examples in $\S 4$. The group involved is $\left(G_{\mathrm{a}}\right)^{6}$. To get Mukai's best example, with the group $\left(G_{\mathrm{a}}\right)^{3}$, to work over the same fields, we can use projective duality, as follows.

Let $q_{1}, \ldots, q_{9}$ be nine distinct points on $\mathbf{P}^{2}$ which are the intersection of two cubics. These points can be represented by a linear map from $A^{9}$ onto $A^{3}$. The kernel has dimension 6 , and so dualizing gives a linear map from $A^{9}$ onto a 6 -dimensional vector space. This gives the dual arrangement of 9 points $p_{1}, \ldots, p_{9}$ in a 5 -dimensional projective space. Dolgachev gives an equivalent description of this arrangement, under our assumption on $q_{1}, \ldots, q_{9}: p_{1}, \ldots, p_{9}$ are the image of $q_{1}, \ldots, q_{9}$ under a Veronese embedding $\mathbf{P}^{2} \rightarrow \mathbf{P}^{5}$ (see [Dol04, Proposition 5.4]).

Corollary 3.3. Let $q_{1}, \ldots, q_{9}$ be nine distinct rational points in $\mathbf{P}^{2}$ over a field $k$ which are the intersection of two cubics. Suppose that no three of $q_{1}, \ldots, q_{9}$ lie on a line. Let $p_{1}, \ldots, p_{9}$ be the dual arrangement of nine points in $\mathbf{P}^{5}$. Then $p_{1}, \ldots, p_{9}$ are in Cremona general position. Therefore, the blow-up $X$ of $\mathbf{P}^{5}$ at $p_{1}, \ldots, p_{9}$ has infinitely generated total coordinate ring, and the corresponding 18-dimensional representation of $\left(G_{\mathrm{a}}\right)^{3}$ over $k$ has infinitely generated ring of invariants.

Proof. Since $q_{1}, \ldots, q_{9}$ are in linear general position in $\mathbf{P}^{2}$, we check by hand that the dual arrangement $p_{1}, \ldots, p_{9}$ is in linear general position in $\mathbf{P}^{5}$. Performing the standard Cremona transformation on $q_{1}, q_{2}, q_{3}$ in $\mathbf{P}^{2}$ and then dualizing corresponds to performing the standard Cremona transformation on $p_{9}, \ldots, p_{4}$ in $\mathbf{P}^{5}$, as Dolgachev-Ortland computed [DO88, Theorem VI.4]. (This is elementary: the assumption that $q_{1}, \ldots, q_{9}$ are the intersection of two cubics is not needed for this duality statement.) Since $q_{1}, \ldots, q_{9}$ are in Cremona general position in $\mathbf{P}^{2}$ by Corollary 3.2, it follows that $p_{1}, \ldots, p_{9}$ are in Cremona general position in $\mathbf{P}^{5}$. Lemma 2.2 gives the rest. 


\section{HILBERT'S 14TH PROBLEM}

Thus, we have counterexamples to Hilbert's 14th problem using an 18-dimensional representation of $\left(G_{\mathrm{a}}\right)^{3}$ over all sufficiently large finite fields and all infinite fields. Finally, let us check that Mukai's final class of examples, using the group $\left(G_{\mathrm{a}}\right)^{4}$, also works over all sufficiently large finite fields and all infinite fields. A net of quadrics in $\mathbf{P}^{3}$ means a linear system $\mathbf{P}^{2}$ of quadrics or, equivalently, a three-dimensional linear subspace of the vector space of quadratic forms in four variables.

Lemma 3.4. Let $p_{1}, \ldots, p_{8}$ be eight distinct points in $\mathbf{P}^{3}$ which are the intersection of three quadrics, $Q_{1} \cap Q_{2} \cap Q_{3}$. Then no four of the points $p_{1}, \ldots, p_{8}$ lie on a plane if and only if every quadric in the net spanned by $Q_{1}, Q_{2}, Q_{3}$ is irreducible.

Proof. If some quadric $Q$ in the net is reducible, then $Q=S_{1} \cup S_{2}$ for some planes $S_{1}$ and $S_{2}$ in $\mathbf{P}^{3}$. Then four of the points $p_{1}, \ldots, p_{8}$ lie on $S_{1}$ (and the other four lie on $S_{2}$ ).

Conversely, suppose that $p_{1}, p_{2}, p_{3}, p_{4}$ lie on a plane $S$ in $\mathbf{P}^{3}$. No three of the points $p_{1}, \ldots, p_{8}$ lie on a line $L$ in $\mathbf{P}^{3}$; otherwise every quadric in the net would contain three points on $L$, hence would contain $L$, contradicting that $Q_{1} \cap Q_{2} \cap Q_{3}=\left\{p_{1}, \ldots, p_{8}\right\}$. So no three of the points $p_{1}, p_{2}, p_{3}, p_{4}$ lie on a line. Therefore, these four points are the complete intersection of two conics in the plane $S$, $p_{1} p_{2} \cup p_{3} p_{4}$ and $p_{1} p_{3} \cup p_{2} p_{4}$. It follows that there is only a pencil (a $\mathbf{P}^{1}$ ) of conics in $S$ through $p_{1}, p_{2}, p_{3}, p_{4}$. Since we have a net $\left(\mathrm{a} \mathbf{P}^{2}\right)$ of quadrics in $\mathbf{P}^{3}$ that contain $p_{1}, p_{2}, p_{3}, p_{4}$, at least one quadric $Q$ in the net must contain the whole plane $S$. Thus, $Q$ is reducible.

Corollary 3.5. Let $p_{1}, \ldots, p_{8}$ be eight distinct points in $\mathbf{P}^{3}$ which are the intersection of three quadrics, and suppose that no four of the points $p_{1}, \ldots, p_{8}$ lie on a plane. Then $p_{1}, \ldots, p_{8}$ are in Cremona general position. Therefore the blow-up $X$ of $\mathbf{P}^{3}$ at $p_{1}, \ldots, p_{8}$ has infinitely generated total coordinate ring, and the corresponding 16-dimensional representation of $\left(G_{\mathrm{a}}\right)^{4}$ over $k$ has infinitely generated ring of invariants.

The first part of Corollary 3.5 was apparently known to Coble [Cob29, §44, last paragraph].

Proof. The Cremona action on $\operatorname{Pic}(X)$ fixes half of the anticanonical class, $-\frac{1}{2} K_{X}=2 H-E_{1}-$ $\cdots-E_{8}$. So performing a standard Cremona transformation on any four of $p_{1}, \ldots, p_{8}$ transforms a quadric through $p_{1}, \ldots, p_{8}$ into a quadric through the new points $p_{1}^{\prime}, \ldots, p_{8}^{\prime}$. Since $p_{1}, \ldots, p_{8}$ are the intersection of three quadrics, $p_{1}^{\prime}, \ldots, p_{8}^{\prime}$ are also the intersection of three quadrics. By Lemma 3.4, since no four of $p_{1}, \ldots, p_{8}$ lie on a plane, all of the quadrics through $p_{1}, \ldots, p_{8}$ are irreducible. Therefore, their proper transforms, the quadrics through $p_{1}^{\prime}, \ldots, p_{8}^{\prime}$, are also all irreducible. So no four of $p_{1}^{\prime}, \ldots, p_{8}^{\prime}$ lie on a plane. Thus, $p_{1}^{\prime}, \ldots, p_{8}^{\prime}$ satisfy the same assumptions as $p_{1}, \ldots, p_{8}$, and so we can repeat the process any number of times. That is, $p_{1}, \ldots, p_{8}$ are in Cremona general position.

\section{Examples over arbitrary fields}

We now prove Theorem 0.1, showing that our three classes of representations with infinitely generated rings of invariants all exist over arbitrary fields. In later sections, we describe the geometry behind these representations in more detail. One benefit will be to construct representations with infinitely generated ring of invariants that are given by simpler formulas. Another benefit will be to prove partial results about exactly when finite generation holds. For now, we just prove Theorem 0.1 as stated.

Theorem 2.1 associates a $2 n$-dimensional representation of $\left(G_{\mathrm{a}}\right)^{n-r}$ over a field $k$ to any arrangement of $n$ distinct $k$-points of $\mathbf{P}^{r-1}$ not contained in a hyperplane. More generally, we can define such a representation associated to a smooth zero-dimensional subscheme of degree $n$ defined over $k$, even if the individual points are not defined over $k$, using the standard technique of 'twisting' 


\section{B. TOTARO}

in Galois cohomology [Ser94, §III.1.3]. Explicitly, given a Galois extension field $K / k$ over which the points are defined, let the Galois group $\operatorname{Gal}(K / k)$ act on $K^{n}$ by permuting the basis vectors as $\operatorname{Gal}(K / k)$ permutes the $n$ points, and by $\sigma(a x)=\sigma(a) \sigma(x)$ for $a \in K$. We can view $K^{n}$ with this Galois action as a 'twisted form' $H$ of the group $\left(G_{\mathrm{a}}\right)^{n}$ over $k$, but it is in fact isomorphic to $\left(G_{\mathrm{a}}\right)^{n}$ over $k$ because $H(k)=\left(K^{n}\right)^{\mathrm{Gal}(K / k)}$ is an $n$-dimensional vector space over $k$. A linear map $K^{n} \rightarrow K^{r}$ associated to the $n$ points in $\mathbf{P}^{r-1}$ is evidently Galois-equivariant, and so (taking Galois invariants) it gives a linear map $H(k) \cong k^{n} \rightarrow k^{r}$. The kernel is a vector space over $k$, which canonically determines an algebraic group $G$ over $k$, clearly isomorphic to $\left(G_{\mathrm{a}}\right)^{n-r}$. Likewise, the $2 n$-dimensional representation of $\left(G_{\mathrm{a}}\right)^{n}$ in Theorem 2.1 gives a $2 n$-dimensional representation of its twisted form $H$ and, hence, of the subgroup $G \cong\left(G_{\mathrm{a}}\right)^{n-r}$ over $k$.

Moreover, whether a ring of invariants $O(V)^{G}$ for a representation $V$ of an algebraic group $G$ is finitely generated does not change under extension of the base field, since the ring of invariants over an extension field $K / k$ is just the ring of invariants over $k$ tensored with $K$. So we can apply the previous section's results. For example, given two plane cubics $C_{1}$ and $C_{2}$ over a field $k$ whose intersection is smooth of dimension zero, consider the associated 18-dimensional representation of $\left(G_{\mathrm{a}}\right)^{6}$ over $k$. If no three points of $C_{1} \cap C_{2}$ over the algebraic closure of $k$ lie on a line, then the corresponding representation of $\left(G_{\mathrm{a}}\right)^{6}$ over $k$ has infinitely generated ring of invariants, by Corollary 3.2. In practice, it is often easier to check the equivalent hypothesis that all cubics in the pencil spanned by $C_{1}$ and $C_{2}$ are irreducible.

For example, consider the cuspidal cubics $(y+z)^{3}+x z^{2}$ and $x^{3}+y^{2} z$ over a field $\mathbf{F}_{p}$. For primes $p \leqslant 23$ (not an optimal bound), we compute that the intersection of these two cubics in $\mathbf{P}^{2}$ is smooth of dimension zero, and that all nonzero linear combinations of these two cubics are irreducible. (This is particularly easy to check for $p=2$ or 3 , where the given cuspidal cubics are the only singular cubics in the pencil.) By Corollary 3.2, for $p \leqslant 23$, the associated 18-dimensional representation of $\left(G_{\mathrm{a}}\right)^{6}$ over $\mathbf{F}_{p}$ has infinitely generated ring of invariants. For fields of characteristic greater than 23 (or characteristic zero), we can apply Corollary 3.5 directly. Namely, the nine points in $\mathbf{P}^{2}$ which form the columns of the following matrix are the base locus of a pencil of cubics, and are in linear general position over $\mathbf{Q}$ and over $\mathbf{F}_{p}$ for all $p>23$ :

$$
\left(\begin{array}{rrrrrrrrr}
1 & 0 & 0 & 1 & 1 & 2 & -3 & -2 & -7 \\
0 & 1 & 0 & 1 & -1 & -1 & 4 & -5 & 2 \\
0 & 0 & 1 & 1 & 2 & 1 & 1 & 1 & -1
\end{array}\right)
$$

(One can look for such examples by choosing eight points in $\mathbf{P}^{2}$ and computing the ninth point on the pencil of cubics through the eight points.) Thus, we have constructed 18-dimensional representations of $\left(G_{\mathrm{a}}\right)^{6}$ with infinitely generated ring of invariants over an arbitrary field.

Now consider again the intersection of the cuspidal cubics $(y+z)^{3}+x z^{2}$ and $x^{3}+y^{2} z$ over a field $\mathbf{F}_{p}$ with $p \leqslant 23$. The projective dual of these nine points in $\mathbf{P}^{2}$ gives a smooth subscheme of degree nine in $\mathbf{P}^{5}$ over $\mathbf{F}_{p}$. Consider the associated representation of $\left(G_{\mathrm{a}}\right)^{3}$ over $\mathbf{F}_{p}$. Since all cubics in this pencil of cubics in $\mathbf{P}^{2}$ are irreducible when $p \leqslant 23$, Corollary 3.3 shows that the ring of invariants for this 18-dimensional representation of $\left(G_{\mathrm{a}}\right)^{3}$ over $\mathbf{F}_{p}$ is infinitely generated. For fields of characteristic greater than 23 or of characteristic zero, we can apply Corollary 3.3 directly, using the previous paragraph's arrangement of nine rational points. Thus, we have constructed 18dimensional representations of $\left(G_{\mathrm{a}}\right)^{3}$ with infinitely generated ring of invariants over an arbitrary field.

We now construct analogous representations of $\left(G_{\mathrm{a}}\right)^{4}$. Over a field $\mathbf{F}_{p}$, consider the quadrics $x y+y^{2}+z^{2}, x w+y^{2}+w^{2}, x z-z w+z^{2}+w^{2}$. We compute that for all primes $p \leqslant 7$ (not an optimal bound), the intersection of these three quadrics is smooth of dimension zero, and all nonzero linear combinations of these quadrics are irreducible. Therefore, for $p \leqslant 7$, the corresponding 


\section{HILBERT'S 14TH PROBLEM}

16-dimensional representation of $\left(G_{\mathrm{a}}\right)^{4}$ over $\mathbf{F}_{p}$ has infinitely generated ring of invariants. For fields of characteristic greater than seven (or characteristic zero), we can apply Corollary 3.5 directly. Namely, the eight points in $\mathbf{P}^{3}$ which form the columns of the following matrix are the base locus of a net of quadrics, and are in linear general position over $\mathbf{Q}$ and over $\mathbf{F}_{p}$ for all $p>7$ :

$$
\left(\begin{array}{rrrrrrrr}
1 & 0 & 0 & 0 & 1 & -2 & 1 & -6 \\
0 & 1 & 0 & 0 & 1 & 2 & -4 & -8 \\
0 & 0 & 1 & 0 & 1 & 1 & -3 & -7 \\
0 & 0 & 0 & 1 & 1 & -3 & 4 & -4
\end{array}\right) .
$$

(One can look for such examples by choosing seven points $p_{1}, \ldots, p_{7}$ in $\mathbf{P}^{3}$ and computing the eighth point on the net of quadrics through $p_{1}, \ldots, p_{7}$.) Thus we have proved the existence of 16 dimensional representations of $\left(G_{\mathrm{a}}\right)^{4}$ with infinitely generated ring of invariants over an arbitrary field, and thus proved Theorem 0.1 .

\section{Elliptic fibrations, and representations of $\left(G_{\mathrm{a}}\right)^{6}$ on $A^{18}$}

We now describe the geometry behind the representations of $\left(G_{\mathrm{a}}\right)^{6}$ in Theorem 0.1. It turns out that finite generation depends on the Mordell-Weil group of a certain elliptic fibration of $\mathbf{P}^{2}$. For a certain class of representations, we can say exactly when the ring of invariants is finitely generated and when it is not. As a concrete application, we give examples of non-finite generation where the coefficients of the representation are very simple (in particular, simpler than the examples in $\S 4$ ). As in $\S 4$, we obtain examples of non-finite generation over all fields, even the field of order two.

The general problem here is to understand the border between finite generation and non-finite generation (for rings of invariants, or for total coordinate rings). Section 3 shows that if we assume a small amount of general position, we obtain examples of non-finite generation. In this section we find that even for some more special arrangements of points, which do not satisfy the hypotheses of $\S 3$, we can still prove non-finite generation. This case of nine points in the plane has been intensely studied, and many of the results of this section can be deduced from various earlier works, as we will see. The main novelty is the precise characterization of finite generation in Theorem 5.2.

Corollary 5.1. Let $\left(G_{\mathrm{a}}\right)^{n}$ act on $V=A^{2 n}$ by

$$
\left(t_{1}, \ldots, t_{n}\right)\left(x_{1}, \ldots, x_{n}, y_{1}, \ldots, y_{n}\right)=\left(x_{1}, \ldots, x_{n}, y_{1}+t_{1} x_{1}, \ldots, y_{n}+t_{n} x_{n}\right) .
$$

Over any field $k$ of characteristic not two or three, consider the subgroup $G=\left(G_{\mathrm{a}}\right)^{6}$ of $\left(G_{\mathrm{a}}\right)^{9}$ which is the kernel of the following linear map $A^{9} \rightarrow A^{3}$ :

$$
\left(\begin{array}{rrrrrrrrr}
-1 & -1 & -1 & 0 & 0 & 0 & 1 & 1 & 1 \\
-1 & 0 & 1 & -1 & 0 & 1 & -1 & 0 & 1 \\
1 & 1 & 1 & 1 & 1 & 1 & 1 & 1 & 1
\end{array}\right)
$$

Consider the restriction of the above 18-dimensional representation of $\left(G_{\mathrm{a}}\right)^{9}$ to the subgroup $\left(G_{\mathrm{a}}\right)^{6}$. The ring of invariants of this representation is not finitely generated over $k$.

There are other (almost equally simple) 18-dimensional representations of $\left(G_{\mathrm{a}}\right)^{6}$ over $\mathbf{F}_{2}$ or $\mathbf{F}_{3}$ for which the ring of invariants is not finitely generated.

Corollary 5.1 is a consequence of the following theorem, which characterizes exactly which intersections of two cubics in $\mathbf{P}^{2}$ yield infinitely generated rings of invariants (or infinitely generated total coordinate rings). We generalize this theorem to all rational elliptic surfaces in Theorem 8.2.

Theorem 5.2. Let $p_{1}, \ldots, p_{9}$ be nine distinct rational points in $\mathbf{P}^{2}$ over a field $k$ which are the intersection of two cubics. Let $a$ be the number of collinear triples of points $p_{1}, \ldots, p_{9}$, let $b$ be 


\section{B. TOTARO}

the number of partitions of $p_{1}, \ldots, p_{9}$ into three collinear triples, and let

$$
\rho=8-a+b .
$$

Then $\rho \geqslant 0$.

If $\rho>0$, then the blow-up $X$ of $\mathbf{P}^{2}$ at $p_{1}, \ldots, p_{9}$ has infinitely generated total coordinate ring, and the corresponding 18-dimensional representation of $\left(G_{\mathrm{a}}\right)^{6}$ over $k$ has infinitely generated ring of invariants.

Conversely, if $\rho=0$, then these rings are finitely generated.

Theorem 5.2 strengthens Corollary 3.2, which proves infinite generation assuming that there are no collinear triples among $p_{1}, \ldots, p_{9}$.

Proof. Let $X$ be the blow-up of $\mathbf{P}^{2}$ at the points $p_{1}, \ldots, p_{9}$. The pencil of cubics through $p_{1}, \ldots, p_{9}$ makes the blow-up $X$ an elliptic surface $f: X \rightarrow \mathbf{P}^{1}$ (or perhaps quasi-elliptic, in characteristics two and three). We want to give criteria for $X$ to contain infinitely many $(-1)$-curves, without requiring that no three of $p_{1}, \ldots, p_{9}$ lie on a line (as in Corollary 3.2). There is, in fact, a complete classification of the intersections of two plane cubics such that the blow-up $X$ contains only finitely many $(-1)$ curves ( $X$ is called an extremal rational elliptic surface), by Miranda-Persson in characteristic zero [MP86] and Lang in positive characteristic [Lan91, Lan94]; see also Cossec-Dolgachev [CD89, $\S 5.6]$. There is also more general work by Nikulin classifying certain types of varieties whose cone of curves is finite polyhedral [Nik00, Nik04]. We do not use any of these classification results, but just explain how to check whether a given rational elliptic surface $X$ has infinitely many $(-1)$-curves.

The $(-1)$-curves in any surface can be described as the smooth rational curves $C$ such that $\left(-K_{X}\right) \cdot C=1$. Since the fibers of the elliptic fibration $f: X \rightarrow \mathbf{P}^{1}$ are in the linear system $\left|-K_{X}\right|$, it follows that the $(-1)$-curves in $X$ are precisely the sections of the elliptic fibration. Thus, to make $X$ have infinitely many $(-1)$-curves, it suffices to arrange that the Mordell-Weil group $\operatorname{Pic}^{0}(E)$ of the general fiber (an elliptic curve over the field $k(t)$ ) has rank at least one. We can define the Mordell-Weil group as the group of sections of this elliptic fibration, with one section considered as the zero section.

For each reducible fiber $F$ of the elliptic fibration $X \rightarrow \mathbf{P}^{1}$, let $r_{F}+1$ be the number of irreducible components of F. By Tate [Tat65, §4.5] and Shioda [Shi72, Corollary 1.5], the Mordell-Weil rank of $E$ over $k(t)$ is equal to $8-\sum r_{F}$. For the reader's convenience, here is a proof. The Picard group of the general fiber $E$ over $k(t)$ is the quotient of the Picard group of the surface $X$ by the classes of all irreducible divisors in $X$ which do not map onto $\mathbf{P}^{1}$. So $\operatorname{Pic}(E)$ is the quotient of $\operatorname{Pic}(X) \cong \mathbf{Z}^{10}$ by the class of $-K_{X}$ (the class of each irreducible fiber of $f: X \rightarrow \mathbf{P}^{1}$ ) and by $r_{F}$ classes for each fiber $F$ with $r_{F}+1$ irreducible components. Moreover, if we write the reducible fibers as $F=\sum_{j=1}^{r_{F}+1} m_{F j} D_{F j}$, then the divisors $D_{F j}$ for all $F$ and all $1 \leqslant j \leqslant r_{F}$, together with $-K_{X}$, are linearly independent in $\operatorname{Pic}(X)_{\mathbf{Q}}$ (even modulo numerical equivalence). This is a fact about any morphism from a surface to a curve [Bea96, pp. 122-123]. Therefore, $\operatorname{Pic}(E)_{\mathbf{Q}}$ has rank $10-1-\sum r_{F}$ and the Mordell-Weil group $\operatorname{Pic}^{0}(E)_{\mathbf{Q}}$ has rank $8-\sum r_{F}$, as required.

The only possible reducible cubics in the given pencil are the sum of a line and a conic, or the sum of three lines. By the proof of Lemma 3.1, the lines occurring in these reducible cubics are precisely the lines through collinear triples among the points $p_{1}, \ldots, p_{9}$. Therefore, $\sum r_{F}$ is equal to the number $a$ of collinear triples, minus the number $b$ of partitions of $p_{1}, \ldots, p_{9}$ into three disjoint collinear triples. (The second term comes in because a cubic in the pencil which consists of three lines contributes only two, not three, to $\sum r_{F}$.) So the Mordell-Weil rank of $E$ over $k(t)$ is $\rho=8-a+b$, which is therefore nonnegative. If $\rho$ is greater than zero, then the blow-up $X$ contains infinitely many $(-1)$-curves. It follows that the total coordinate ring of $X$ is infinitely generated, 
and that the corresponding ring of invariants for an 18-dimensional representation of $\left(G_{\mathrm{a}}\right)^{6}$ over $k$ is infinitely generated.

It remains to show that when $\rho=0$, then the total coordinate ring of $X$ is finitely generated. Our main tools will be the cone and contraction theorems, proved for log surfaces in any characteristic by Kollár and Kovács [KK94, Theorems 2.1.1 and 2.3.3], and $\mathrm{Hu}$ and Keel's notion of a Mori dream space [HK00]. In particular, for any klt pair $(X, \Delta)$ of dimension two, we can contract any $\left(K_{X}+\Delta\right)$-negative extremal face of the closed cone of curves $\overline{N E}(X)$, yielding a projective variety. Kollár and Kovács only state this for contractions of extremal rays, but the result for higherdimensional extremal faces is a consequence. The point is that for any $\left(K_{X}+\Delta\right)$-negative extremal face $F$, if the contraction $f: X \rightarrow Y$ of one ray of $F$ is birational, then the pair $\left(Y, f_{*} \Delta\right)$ is klt and the image of $F$ is a $\left(K_{Y}+f_{*} \Delta\right)$-negative extremal face [KK94, Lemma 2.3.5]. Then the cone theorem (including the contraction theorem) for higher-dimensional extremal faces follows by induction on the Picard number of $X$.

The assumption $\rho=0$ tells us that the subspace $\left(K_{X}\right)^{\perp} \cong \mathbf{Q}^{9}$ of $\operatorname{Pic}(X) \otimes \mathbf{Q}$ is spanned by the curves in $X$ which are contained in fibers of the morphism $X \rightarrow \mathbf{P}^{1}$. The sum of all of the curves in a reducible fiber, with multiplicities, is numerically equivalent to a general fiber $-K_{X}$; so $\left(K_{X}\right)^{\perp}$ is spanned just by the finitely many irreducible components of reducible fibers of $X \rightarrow \mathbf{P}^{1}$. We can also describe these curves as the $(-2)$-curves on $X$ (curves isomorphic to $\mathbf{P}^{1}$ with self-intersection -2 ), using the fact that $-K_{X}$ has degree zero on any $(-2)$-curve.

Another consequence of the assumption $\rho=0$ is that the Mordell-Weil rank of the elliptic surface $X \rightarrow \mathbf{P}^{1}$ is zero. Since the sections of $X \rightarrow \mathbf{P}^{1}$ are exactly the $(-1)$-curves, this means that $X$ contains only finitely many $(-1)$-curves. By the cone theorem, the extremal rays of the closed cone of curves in the $K_{X}$-negative half space $\left(K_{X}\right)^{<0}$ are all spanned by $(-1)$-curves, and so there are only finitely many extremal rays in $\left(K_{X}\right)^{<0}$. Since $-K_{X}$ is semi-ample (corresponding to the contraction $\left.X \rightarrow \mathbf{P}^{1}\right)$, we know that the closed cone of curves is contained in $\left(K_{X}\right)^{\leqslant 0}$.

Moreover, for any curve $C$ on $X$ which is not one of the finitely many (-2)-curves $D_{F j}$, we have $C \cdot D_{F j} \geqslant 0$ for all $F$ and $j$. As the curves $D_{F j}$ span $\left(K_{X}\right)^{\perp}$ and are divided into subsets with $\sum_{j} m_{F j} D_{F j} \sim-K_{X}$, where $m_{F j}>0$, the cone

$$
\left\{x \in N^{1}(X): x \cdot D_{F j} \geqslant 0 \text { for all } F, j\right\}
$$

is a finite polyhedral subcone of $\left(K_{X}\right)^{\leqslant 0}$ whose intersection with $\left(K_{X}\right)^{\perp}$ is just $\mathbf{R}^{\geqslant 0} \cdot\left(-K_{X}\right)$. Since all curves except the $(-2)$-curves belong to this cone, it follows that the closed cone of curves is finite polyhedral, spanned by the $(-1)$-curves and $(-2)$-curves. (This consequence of $\rho=0$ was proved already by Nikulin [Nik00, Example 1.4.1].)

By a result of $\mathrm{Hu}$ and Keel, a projective variety has finitely generated total coordinate ring if and only if it is a 'Mori dream space' [HK00, Proposition 2.9]. For a smooth projective surface, this means that the first Betti number is zero, the closed cone of curves is finite polyhedral, and every codimension-one face of the cone can be contracted.

Thus, it remains to show that every codimension-one face of the closed cone of curves $\overline{N E}(X)$ can be contracted. A face in the $K_{X}$-negative half space (equivalently, a face spanned by $(-1)$ curves) can be contracted by the cone theorem. On the other hand, because the $(-2)$-curves span the hyperplane $\left(-K_{X}\right)^{\perp}$, the intersection $\overline{N E}(X) \cap\left(K_{X}\right)^{\perp}$ is a codimension-one face (the span of all of the $(-2)$-curves). We can contract this face using the line bundle $-K_{X}$, corresponding to the contraction $X \rightarrow \mathbf{P}^{1}$.

It remains to contract a codimension-one face $A$ of $\overline{N E}(X)$ spanned by some $(-1)$-curves and some $(-2)$-curves. Let $C_{1}, \ldots, C_{r}$ be the $(-2)$-curves in the face $A$. These cannot include all of the irreducible components of any fiber $F$ of $X \rightarrow \mathbf{P}^{1}$, because then $-K_{X} \sim \sum_{j} m_{F j} D_{F j}$ would belong to the face $A$. However, $-K_{X}$ is a positive linear combination of all of the $(-2)$-curves. 


\section{B. TOTARO}

Therefore, $-K_{X}$ belongs to the interior of the codimension-one face $\left(K_{X}\right)^{\perp} \cap \overline{N E}(X)$, and so it cannot belong to any other face.

Since the (-2)-curves $C_{1}, \ldots, C_{r}$ do not include all of the irreducible components of any fiber of $X \rightarrow \mathbf{P}^{1}$, the intersection pairing on $\bigoplus_{i=1}^{r} \mathbf{Q} C_{i}$ is negative definite [Bea96, pp. 122-123]. So there are rational numbers $a_{i}$ such that $\left(\sum a_{i} C_{i}\right) \cdot C_{j}=-1$ for all $j=1, \ldots, r$. By Kollár-Mori [KM98, Lemma 3.41], it follows that $a_{i} \geqslant 0$ for all $i$.

Let $\Delta=\epsilon \sum a_{i} C_{i}$ for some small positive number $\epsilon$. The pair $(X, \Delta)$ is klt [KK94, Definition 2.2.6] since $X$ is smooth and $\epsilon$ is small; so we can apply the contraction theorem. Clearly $K_{X}+\Delta$ is negative on the (-1)-curves $C$ in the face $A$, since $K_{X} \cdot C=-1$ and $\epsilon$ is small. Also, $K_{X}+\Delta$ has degree $-\epsilon$ on the $(-2)$-curves $C_{1}, \ldots, C_{r}$ in $A$. Thus, the face $A$ is $\left(K_{X}+\Delta\right)$-negative. By the cone theorem, we can contract the face $A$. This completes the proof that the total coordinate ring of $X$ is finitely generated.

Proof of Corollary 5.1. For any field $k$ of characteristic not two or three, consider the nine points in $\mathbf{P}^{2}(k)$ given in affine coordinates by $(x, y)$ where $x$ and $y$ run through the set $\{-1,0,1\}$. These points are the intersection of the two cubics $x^{3}=x z^{2}$ and $y^{3}=y z^{2}$, each consisting of three lines through a point in $\mathbf{P}^{2}$. We count (using the assumption on the characteristic) that there are eight collinear triples among these points $p_{1}, \ldots, p_{9}$, and two partitions of $p_{1}, \ldots, p_{9}$ into three collinear triples. (The two partitions into three collinear triples correspond to two cubics $x^{3}-x z^{2}=x(x-z)(x+z)$ and $y^{3}-y z^{2}=y(y-z)(y+z)$ in our pencil which are unions of three lines, and the other two collinear triples correspond to two other reducible cubics in the pencil, $\left(x^{3}-x z^{2}\right)-\left(y^{3}-y z^{2}\right)=$ $(x-y)\left(x^{2}+x y+y^{2}-z^{2}\right)$ and $\left(x^{3}-x z^{2}\right)+\left(y^{3}-y z^{2}\right)=(x+y)\left(x^{2}-x y+y^{2}-z^{2}\right)$.) So $\rho=8-8+2=2$ is greater than zero. By Theorem 5.2, the blow-up $X$ of $\mathbf{P}^{2}$ at this set of nine points has infinitely many (-1)-curves. Thus, we obtain an 18-dimensional representation of $\left(G_{\mathrm{a}}\right)^{6}$ over $\mathbf{F}_{p}$ for $p \geqslant 5$ (or over $\mathbf{Q}$ ) whose ring of invariants is not finitely generated. More precisely, the Mordell-Weil group has rank two in this case.

We refer to Appendix A for simple examples of 18-dimensional representations of $\left(G_{\mathrm{a}}\right)^{6}$ over $\mathbf{F}_{2}$ or $\mathbf{F}_{3}$ whose rings of invariants are not finitely generated.

\section{A new fibration of $\mathrm{P}^{5}$ by abelian surfaces, and representations of $\left(G_{\mathrm{a}}\right)^{3}$ on $A^{18}$}

We now give a richer geometric explanation for the infinitely generated rings of invariants for $\left(G_{\mathrm{a}}\right)^{3}$ constructed in Corollary 3.3: they are explained by a fibration of a blow-up of $\mathbf{P}^{5}$ by abelian surfaces.

Theorem 6.1. Let $q_{1}, \ldots, q_{9}$ be nine distinct rational points in $\mathbf{P}^{2}$ over a field $k$ which are the intersection of two cubics. Suppose that no three of $p_{1}, \ldots, p_{9}$ lie on a line. Let $p_{1}, \ldots, p_{9}$ be the dual arrangement of nine points in $\mathbf{P}^{5}$. Then the blow-up $X$ of $\mathbf{P}^{5}$ at $p_{1}, \ldots, p_{9}$ is pseudo-isomorphic to a smooth projective variety $W$ which is an abelian surface fibration over $\mathbf{P}^{3}$ with a section. The Mordell-Weil group of $W$ over $\mathbf{P}^{3}$ has rank eight. The translates by the Mordell-Weil group of the (-1)-divisors $E_{1}, \ldots, E_{9}$ yield infinitely many $(-1)$-divisors on $W$ or, equivalently, on $X$. As a result, the total coordinate ring of $X$ is not finitely generated, and the corresponding 18-dimensional representation of $\left(G_{\mathrm{a}}\right)^{3}$ over $k$ has infinitely generated ring of invariants.

Proof. We recall Dolgachev's equivalent description of the dual arrangement, under our assumption on $q_{1}, \ldots, q_{9}: p_{1}, \ldots, p_{9}$ are the image of $q_{1}, \ldots, q_{9}$ under a Veronese embedding $\mathbf{P}^{2} \rightarrow \mathbf{P}^{5}$ (see [Dol04, Proposition 5.4]).

There is a natural homomorphism from the group $\mathbf{Z}^{8} \rtimes \mathbf{Z} / 2$ to the automorphism group of the blow-up $Y$ of $\mathbf{P}^{2}$ at $q_{1}, \ldots, q_{9}$ (see [DO88, p. 124]). Explicitly, $Y$ is an elliptic surface, with the $\mathbf{Z}^{8}$ subgroup giving translations by differences of sections, and the $\mathbf{Z} / 2$ giving the map $z \mapsto-z$ with 
respect to some zero-section. The construction of $p_{1}, \ldots, p_{9}$ by duality gives a corresponding action of $\mathbf{Z}^{8} \rtimes \mathbf{Z} / 2$ by pseudo-automorphisms of the blow-up $X$ of $\mathbf{P}^{5}$ at $p_{1}, \ldots, p_{9}$, by Dolgachev [Dol04, $\S 5.12]$. Moreover, these pseudo-automorphisms are defined on the blown-up Veronese surface $Y$ inside $X$ and give the action we mentioned of $\mathbf{Z}^{8} \rtimes \mathbf{Z} / 2$ on $Y$. Our goal is to give a more geometric interpretation of these pseudo-automorphisms of $X$.

The linear system of $-\frac{1}{2} K_{X}=3 H-2 E_{1}-\cdots-2 E_{9}$ (that is, cubics on $\mathbf{P}^{5}$ singular at $p_{1}, \ldots, p_{9}$ ) is a $\mathbf{P}^{3}$, with base locus a union of 45 curves: the 36 lines through pairs of points $p_{i}$ and the nine rational normal curves through any eight of the points $p_{1}, \ldots, p_{9}$. Indeed, the base locus of the linear system $\left|-\frac{1}{2} K_{X}\right|$, viewed on $\mathbf{P}^{5}$, is an intersection of four cubics. One checks that the base locus has dimension one, which is easy in a particular example (and therefore holds for a general 9 -tuple $p_{1}, \ldots, p_{9}$ as above). Thus, the base locus is a complete intersection of four cubics in $\mathbf{P}^{5}$ and hence has degree 81 . The 45 curves mentioned have total degree 81 , and they are all in the base locus, because $-\frac{1}{2} K_{X}$ has degree -1 on these curves. So the base locus is precisely the union of these 45 curves, each with multiplicity one.

We can describe the rational map $X \rightarrow \mathbf{P}^{3}$ associated to $-\frac{1}{2} K_{X}$. The base locus of this linear system consists of 45 disjoint curves isomorphic to $\mathbf{P}^{1}$ in $X$, all with normal bundle $O(-1)^{\oplus 4}$. Using the linear system $\left|-\frac{1}{2} K_{X}\right|$, we can perform an inverse flip on these 45 curves, giving another smooth projective five-fold $W$ in which the $45 \mathbf{P}^{1}$ with normal bundle $O(-1)^{\oplus 4}$ have been replaced by $\mathbf{P}^{3}$ with normal bundle $O(-1)^{\oplus 2}$. Explicitly, $W$ can be described as Proj of the $\operatorname{ring} R(X, L)=$ $\bigoplus_{a \geqslant 0} H^{0}(X, a L)$ for any line bundle $L$ which is a positive linear combination of $H+5\left(-\frac{1}{2} K_{X}\right)$ and $-\frac{1}{2} K_{X}$. On this pseudo-isomorphic variety $W$, the linear system $\left|-\frac{1}{2} K_{W}\right|\left(=\left|-\frac{1}{2} K_{X}\right|\right)$ is basepointfree, giving a morphism $W \rightarrow \mathbf{P}^{3}$. The generic fiber of $W \rightarrow \mathbf{P}^{3}$ is a principally polarized abelian surface. The (-1)-divisors $E_{1}, \ldots, E_{9}$ on $W$ all induce the same polarization of the generic fiber, which is two times a principal polarization. (Geometrically, each divisor $E_{i}$ intersects the general fiber in a curve of genus five.) The $45 \mathbf{P}^{3}$ we have produced in $W$ are sections of the fibration, and their differences generate only a rank-eight subgroup of the Mordell-Weil group, for $p_{1}, \ldots, p_{9}$ general as above.

To check that the smooth fibers of $W \rightarrow \mathbf{P}^{3}$ are abelian surfaces, note that the canonical bundle $K_{W}$ is trivial on all fibers, since $-\frac{1}{2} K_{W}$ is pulled back from $\mathbf{P}^{3}$. So the smooth fibers have trivial canonical bundle and hence (say, in characteristic not two or three) are either K3 surfaces or abelian surfaces. To see that they are abelian surfaces, it suffices to compute that $c_{2}(W)\left(-\frac{1}{2} K_{W}\right)^{3}=0$, since K3 surfaces have Euler characteristic $c_{2}$ equal to 24 while abelian surfaces have $c_{2}=0$. To do that calculation, one can compute that $c_{2}(X)\left(-\frac{1}{2} K_{X}\right)^{3}=45$ and that performing an inverse flip (replacing a $\mathbf{P}^{1}$ with normal bundle $O(-1)^{\oplus 4}$ by a $\mathbf{P}^{3}$ with normal bundle $O(-1)^{\oplus 2}$ ) lowers $c_{2}(X)\left(-\frac{1}{2} K_{X}\right)^{3}$ by one.

The following result helps to show what is going on.

Lemma 6.2. Let $f: X \rightarrow S$ be a proper morphism of smooth varieties over a field such that $K_{X}$ has degree zero on all curves $C$ with $f(C)=$ point. Suppose that the smooth locus of the generic fiber has a group structure (for example, the generic fiber could be an abelian variety, or a cuspidal cubic curve in characteristic two or three). Then for any sections $S_{1}$ and $S_{2}$ of $f$ over the generic point of $S$, there is a pseudo-automorphism of $X$ over $S$ that maps $S_{1}$ to $S_{2}$.

Proof. We use a basic fact of minimal model theory: for any proper morphisms $f_{i}: X_{i} \rightarrow S$, $i=1,2$, such that $X_{i}$ is smooth and $K_{X_{i}}$ has nonnegative degree on all curves that map to a point in $S$, every birational map from $X_{1}$ to $X_{2}$ over $S$ is a pseudo-isomorphism. The proof works in any characteristic [KM98, Theorem 3.52], and applies more generally to varieties with terminal singularities. Any section of the morphism $f: X \rightarrow S$ over the generic point of $S$ automatically lies in the smooth locus of the generic fiber, which we assume has a group structure. Therefore, 


\section{B. TOTARO}

for any sections $S_{1}$ and $S_{2}$ over the generic point, adding $S_{2}-S_{1}$ using the group structure is a pseudo-automorphism of $X$ over $S$.

Since the Mordell-Weil group of the generic fiber of $W \rightarrow \mathbf{P}^{3}$ has rank eight, it is in particular infinite. By the fact of minimal model theory just stated, the Mordell-Weil group acts on $W$ or, equivalently, on $X$, by pseudo-automorphisms. These pseudo-automorphisms of $X$ map the $(-1)$ divisor $E_{1}$ to infinitely many other $(-1)$-divisors. It follows that the total coordinate ring of $X$ is not finitely generated, and that the corresponding representation of $\left(G_{\mathrm{a}}\right)^{3}$ has infinitely generated ring of invariants, as we already knew from Corollary 3.3.

\section{An elliptic fibration of $\mathrm{P}^{3}$, and representations of $\left(G_{\mathrm{a}}\right)^{4}$ on $A^{16}$}

We now describe the geometry behind the representations of $\left(G_{\mathrm{a}}\right)^{4}$ in Theorem 0.1 in more detail. As in Theorem 5.2, the key concept is the Mordell-Weil group of an elliptic fibration, in this case an elliptic fibration of a blow-up of $\mathbf{P}^{3}$. For a certain class of representations, we can show that the ring of invariants is infinitely generated except in a very special situation. As a concrete application, we give examples of non-finite generation where the coefficients of the representation are very simple (in particular, simpler than the examples in $\S 4$ ). As in $\S 4$, we obtain examples of non-finite generation over all fields, even the field of order two.

COROLlary 7.1. Over any field $k$ of characteristic not two, start with Nagata's representation of $\left(G_{\mathrm{a}}\right)^{8}$ on $A^{16}$ as in Theorem 2.1, and restrict to the subgroup $\left(G_{\mathrm{a}}\right)^{4}$ spanned by the rows of the following $4 \times 8$ matrix:

$$
\left(\begin{array}{llllllll}
0 & 0 & 0 & 0 & 1 & 1 & 1 & 1 \\
0 & 0 & 1 & 1 & 0 & 0 & 1 & 1 \\
0 & 1 & 0 & 1 & 0 & 1 & 0 & 1 \\
1 & 1 & 1 & 1 & 1 & 1 & 1 & 1
\end{array}\right) .
$$

Then the ring of invariants is not finitely generated over $k$. There are other (almost equally simple) 16-dimensional representations of $\left(G_{\mathrm{a}}\right)^{4}$ over $\mathbf{F}_{2}$ for which the ring of invariants is not finitely generated.

Corollary 7.1 is a consequence of the following theorem. Combined with Prendergast-Smith's results discussed below, Theorem 7.2 characterizes exactly which intersections of three quadrics in $\mathbf{P}^{3}$ yield infinitely generated rings of invariants (or infinitely generated total coordinate rings).

Theorem 7.2. Let $p_{1}, \ldots, p_{8}$ be eight distinct rational points in $\mathbf{P}^{3}$ over a field $k$ which are the intersection of three quadrics. Let a be the number of coplanar quadruples of points $p_{1}, \ldots, p_{8}$, and define

$$
\rho=7-\frac{a}{2}
$$

Then $\rho \geqslant 0$. If $\rho$ is greater than zero, then the blow-up $X$ of $\mathbf{P}^{3}$ at $p_{1}, \ldots, p_{8}$ has infinitely generated total coordinate ring, and the corresponding 16-dimensional representation of $\left(G_{\mathrm{a}}\right)^{4}$ over $k$ has infinitely generated ring of invariants.

Theorem 7.2 strengthens Corollary 3.5, which proves infinite generation assuming that there are no coplanar quadruples among $p_{1}, \ldots, p_{8}$. Prendergast-Smith has classified the intersections of three quadrics in $\mathbf{P}^{3}$ with $\rho=0$ over an arbitrary field, and strengthened Theorem 7.2 to show that finite generation holds if and only if $\rho=0$ (see [Pre08]).

Proof. It is classical that, after blowing up eight points in $\mathbf{P}^{3}$ which are a complete intersection of three quadrics, we have an elliptic fibration $f: X \rightarrow \mathbf{P}^{2}$; a suitable reference is DolgachevOrtland [DO88, Theorem VI.9]. Let us prove this. We have a net of quadrics through the given 
eight points. So the vector space of sections of $-\frac{1}{2} K_{X}=2 H-E_{1}-\cdots-E_{8}$ has dimension three, and it gives a rational map $f$ from $X$ to $\mathbf{P}^{2}$ which is clearly defined outside $E_{1}, \ldots, E_{8}$. As our three quadrics intersect in eight distinct points, their intersection must be transverse at each of the eight points, and so $f$ is in fact a morphism $X \rightarrow \mathbf{P}^{2}$, with $f^{*} H \cong-\frac{1}{2} K_{X}$. It follows that the canonical bundle $K_{X}$ is trivial on all fibers of $f$, and so all smooth fibers are curves with trivial canonical bundle, that is, elliptic curves. (Explicitly, all fibers are complete intersections of two quadrics in $\mathbf{P}^{3}$.) This construction also shows that the exceptional divisors $E_{1}, \ldots, E_{8}$ are sections of $f$.

By Lemma 6.2, for any two sections $S_{1}$ and $S_{2}$ of the minimal elliptic fibration $f$ over the general point of $\mathbf{P}^{2}$, adding $S_{2}-S_{1}$ using the group structure on the general fiber is a pseudo-automorphism of $X$ which takes $S_{1}$ to $S_{2}$. Since the exceptional divisor $E_{1}$ is a section of $f$ and also a (-1)-divisor in Mukai's sense, it follows that all sections of $f$ over the generic point of $Y$ are (-1)-divisors in $X$.

Thus, suppose that we can check that the general fiber $E$ over $k\left(\mathbf{P}^{2}\right)$ of our elliptic fibration has infinite Mordell-Weil group. Then $X$ has infinitely many (-1)-divisors, and so the total coordinate ring of $X$ is not finitely generated. Equivalently, the ring of invariants for the 16-dimensional representation of $\left(G_{\mathrm{a}}\right)^{4}$ we are considering is not finitely generated.

The Picard group of the general fiber $E$ over the function field $k\left(\mathbf{P}^{2}\right)$ is the quotient of the Picard group of the three-fold $X$ by the classes of all irreducible divisors in $X$ which do not map onto $\mathbf{P}^{2}$. Since $f^{*}(H)=-\frac{1}{2} K_{X}$ and $\operatorname{Pic}\left(\mathbf{P}^{2}\right)=\mathbf{Z} H$, the pullback under $f: X \rightarrow \mathbf{P}^{2}$ of every irreducible divisor in $\mathbf{P}^{2}$ is a multiple of $-\frac{1}{2} K_{X}$. So $\operatorname{Pic}(E)$ is the quotient of $\operatorname{Pic}(X) \cong \mathbf{Z}^{9}$ by the class of $-\frac{1}{2} K_{X}$ together with $r_{F}$ classes for each irreducible divisor in $\mathbf{P}^{2}$ whose inverse image in $X$ has $r_{F}+1$ irreducible components, say $\sum_{j=1}^{r_{F}+1} m_{F j} D_{F j}$. Moreover, the divisors $D_{F j}$ for all $F$ and $1 \leqslant j \leqslant r_{F}$, together with $-\frac{1}{2} K_{X}$, are linearly independent in $\operatorname{Pic}(X)_{\mathbf{Q}}$ (even modulo numerical equivalence). This follows from the corresponding fact about morphisms from a surface to a curve [Bea96, pp. 122$123]$ by restricting the morphism $X \rightarrow \mathbf{P}^{2}$ to the inverse image of a general line in $\mathbf{P}^{2}$. Therefore $\operatorname{Pic}(E)_{\mathbf{Q}}$ has rank $9-1-\sum r_{F}$, and the Mordell-Weil group $\operatorname{Pic}^{0}(E)_{\mathbf{Q}}$ has rank $7-\sum r_{F}$.

Let us analyze the subset of $\mathbf{P}^{2}$ over which $f: X \rightarrow \mathbf{P}^{2}$ has reducible fibers. Suppose that some fiber (the intersection of two quadrics $Q_{1}$ and $Q_{2}$ in our net) contains a line $L$. Let $Q_{3}$ be a quadric in our net which is not in the pencil spanned by $Q_{1}$ and $Q_{2}$. Since $Q_{1} \cap Q_{2} \cap Q_{3}=\left\{p_{1}, \ldots, p_{8}\right\}$ is smooth of dimension zero, $Q_{3}$ must intersect $L$ transversely in two points; so $L$ must be the line through two of the points $p_{1}, \ldots, p_{8}$. Since $Q_{3}$ does not contain $L$, the pencil spanned by $Q_{1}$ and $Q_{2}$ is the unique pencil in our net whose base locus contains $L$. Thus, there are at most $\left(\begin{array}{l}8 \\ 2\end{array}\right)=28$ fibers of $\mathbf{P}^{3} \rightarrow \mathbf{P}^{2}$ that contain a line.

So, apart from finitely many fibers, every fiber $F$ of $\mathbf{P}^{3} \rightarrow \mathbf{P}^{2}$ must be a union of irreducible curves of degree at least two, with some multiplicities, and with total degree four. The only reducible possibility is for $F$ to be a union of two irreducible conic curves (curves of degree two), each with multiplicity one. Since every conic in $\mathbf{P}^{3}$ is contained in a plane, $F$ is contained (at least as a set) in a reducible quadric $Q_{1}$, the union of the two planes. Since the fiber $F$ contains $\left\{p_{1}, \ldots, p_{8}\right\}, Q_{1}$ belongs to the net of quadrics through $\left\{p_{1}, \ldots, p_{8}\right\}$. It is clear that the intersection of $Q_{1}$ with every other quadric in our net is reducible. Equivalently, $Q_{1}$ corresponds to a line in $\mathbf{P}^{2}$ over which every fiber of $X \rightarrow \mathbf{P}^{2}$ is reducible.

Thus, we find something not at all clear a priori: the elliptic fibration $X \rightarrow \mathbf{P}^{2}$ has reducible fibers over the union of a finite set and finitely many lines. These lines are in one-to-one correspondence with the reducible quadrics through $p_{1}, \ldots, p_{8}$. Over a general point of each line, the fiber has exactly two irreducible components. Thus, the number $\sum r_{F}$ is equal to the number of reducible quadrics through $p_{1}, \ldots, p_{8}$.

Since a reducible quadric is the union of two planes, the number $\sum r_{F}$ is one half the number of coplanar quadruples among $p_{1}, \ldots, p_{8}$. (Recall from the proof of Lemma 3.4 that $p_{1}, \ldots, p_{4}$ are 


\section{B. TOTARO}

coplanar if and only if $p_{5}, \ldots, p_{8}$ are coplanar.) Thus, the Mordell-Weil rank of the elliptic fibration $X \rightarrow \mathbf{P}^{2}$ over the generic point of $\mathbf{P}^{2}$ is $\rho=7-a / 2$. Therefore, $\rho$ is nonnegative. If $\rho$ is greater than zero, then the Mordell-Weil group is infinite, $X$ contains infinitely many ( -1$)$-divisors, and the total coordinate ring of $X$ and the corresponding ring of invariants are infinitely generated.

Proof of Corollary 7.1. Let $k$ be any field of characteristic not two. By Theorem 2.1, the ring of invariants in Corollary 7.1 is the total coordinate ring of the blow-up $X$ of $\mathbf{P}^{3}$ at eight points, namely the eight points given in affine coordinates $w=1$ by $(x, y, z)$ with $x, y, z \in\{0,1\}$. These eight points are the intersection of three (very simple) quadric surfaces, $x^{2}=x w, y^{2}=y w$, and $z^{2}=z w$. To be precise, it might appear that the above representation of $\left(G_{\mathrm{a}}\right)^{4}$ corresponds to the dual of this arrangement of eight points in $\mathbf{P}^{3}$, but in fact the dual arrangement is projectively isomorphic to the given one. That is a general property of complete intersections of 3 quadrics in $\mathbf{P}^{3}$, by Coble [Cob29] and Dolgachev-Ortland [DO88, Theorem III.3, Example III.6].

Using that the field $k$ has characteristic not two, we count that there are exactly 12 coplanar quadruples among these eight points, corresponding to six reducible quadrics in our net: $x(x-w)$, $y(y-w), z(z-w),\left(x^{2}-x w\right)-\left(y^{2}-y w\right)=(x-y)(x+y-w),(x-z)(x+z-w)$, and $(y-z)(y+z-w)$. Since $\rho=7-\frac{1}{2}(12)=1$ is greater than zero, Theorem 7.2 shows that the Mordell-Weil group of the general fiber $E$ of $X$ over $k\left(\mathbf{P}^{2}\right)$ is infinite, and therefore the ring of invariants for the given 16-dimensional representation of $\left(G_{\mathrm{a}}\right)^{4}$, over $\mathbf{F}_{p}$ for $p \geqslant 3$ or over $\mathbf{Q}$, is not finitely generated.

Appendix A gives a simple example of a 16-dimensional representation of $\left(G_{\mathrm{a}}\right)^{4}$ over $\mathbf{F}_{2}$ whose ring of invariants is not finitely generated. Corollary 7.1 is thus proved.

\section{A generalization of the Kawamata and Morrison conjectures on Calabi-Yau varieties}

Theorems 5.2 and 7.2 relate finite generation of the total coordinate ring, in some situations, to finiteness of a certain Mordell-Weil group. In this section, we make a more general conjecture: the cone of curves of any variety with semi-ample anticanonical bundle should be controlled by a certain group, which may be infinite. (A line bundle is semi-ample if some positive multiple is basepointfree.) This would follow from a generalization of the conjectures of Kawamata and Morrison on Calabi-Yau fiber spaces to allow klt pairs (Conjecture 8.1). We prove the conjecture for smooth projective surfaces with semi-ample anticanonical bundle, the new case being that of rational elliptic surfaces (Theorem 8.2).

For a projective morphism $f: X \rightarrow S$ of normal varieties with connected fibers, define $N^{1}(X / S)$ as the real vector space spanned by Cartier divisors on $X$ modulo numerical equivalence on curves on $X$ mapped to a point in $S$. Define a small Q-factorial modification (SQM) of $X$ over $S$ to be a birational map $f: X \rightarrow X^{\prime}$ over $S$, with $X^{\prime}$ projective over $S$ and Q-factorial, which is an isomorphism in codimension one. A Cartier divisor $D$ on $X$ is called $f$-nef (respectively $f$-movable, $f$-effective) if $D \cdot C \geqslant 0$ for every curve $C$ on $X$ which is mapped to a point in $S$ (respectively, if $\operatorname{codim}\left(\operatorname{supp}\left(\operatorname{coker}\left(f^{*} f_{*} O_{X}(D) \rightarrow O_{X}(D)\right)\right)\right) \geqslant 2$, if $\left.f_{*} O_{X}(D) \neq 0\right)$. For an R-divisor $\Delta$ on a Q-factorial variety $X$, the pair $(X, \Delta)$ is $k l t$ if, for any resolution $\pi: \widetilde{X} \rightarrow X$ with a simple normal crossing R-divisor $\widetilde{\Delta}$ such that $K_{\widetilde{X}}+\widetilde{\Delta}=\pi^{*}\left(K_{X}+\Delta\right)$, the coefficients of $\widetilde{\Delta}$ are less than one [KM98, Definition 2.34].

The $f$-nef cone $\bar{A}(X / S)$ (respectively the closed $f$-movable cone $\bar{M}(X / S)$ ) is the closed convex cone in $N^{1}(X / S)$ generated by the numerical classes of $f$-nef divisors (respectively $f$-movable divisors). The $f$-effective cone $B^{e}(X / S)$ is the convex cone, not necessarily closed, generated by $f$-effective Cartier divisors. We call $A^{e}(X / S)=\bar{A}(X / S) \cap B^{e}(X / S)$ and $M^{e}(X / S)=\bar{M}(X / S) \cap$ $B^{e}(X / S)$ the $f$-effective $f$-nef cone and the $f$-effective $f$-movable cone, respectively. Finally, a finite 


\section{HILBERT'S 14TH PROBLEM}

rational polyhedral cone in $N^{1}(X / S)$ means the closed convex cone spanned by a finite set of Cartier divisors on $X$.

Conjecture 8.1. Let $f: X \rightarrow S$ be a projective morphism with connected fibers, $(X, \Delta)$ a $\mathbf{Q}$ factorial klt pair with $\Delta$ effective. Suppose that $K_{X}+\Delta$ is numerically trivial over $S$. Let Aut $(X / S)$ and $\operatorname{PsAut}(X / S)$ denote the groups of automorphisms or pseudo-automorphisms of $X$ over the identity on $S$. Then we have the following.

(1) The number of $\operatorname{Aut}(X / S)$-equivalence classes of faces of the cone $A^{e}(X / S)$ corresponding to birational contractions or fiber space structures is finite. Moreover, there exists a finite rational polyhedral cone $\Pi$ which is a fundamental domain for the action of $\operatorname{Aut}(X / S)$ on $A^{e}(X / S)$ in the sense that:

(a) $A^{e}(X / S)=\bigcup_{g \in \operatorname{Aut}(X / S)} g_{*} \Pi$;

(b) Int $\Pi \cap g_{*} \operatorname{Int} \Pi=\emptyset$ unless $g_{*}=1$.

(2) The number of $\operatorname{PsAut}(X / S)$-equivalence classes of chambers $A^{e}\left(X^{\prime} / S, \alpha\right)$ on the cone $M^{e}(X / S)$ corresponding to marked SQMS $X^{\prime} \rightarrow S$ of $X \rightarrow S$ is finite. Equivalently, the number of isomorphism classes over $S$ of $S Q M$ s of $X$ over $S$ (ignoring the birational identification with $X)$ is finite. Moreover, there exists a finite rational polyhedral cone $\Pi^{\prime}$ which is a fundamental domain for the action of $\operatorname{PsAut}(X / S)$ on $M^{e}(X / S)$.

Note that Conjecture 8.1 would not be true for Calabi-Yau pairs (pairs $(X, \Delta)$ with $K_{X}+\Delta \equiv 0$ ) that are log-canonical (or dlt) rather than klt. Let $X$ be the blow-up of $\mathbf{P}^{2}$ at nine very general points. Let $\Delta$ be the proper transform of the unique smooth cubic curve through the nine points; then $K_{X}+\Delta \equiv 0$, and so $(X, \Delta)$ is a log-canonical Calabi-Yau pair. The surface $X$ contains infinitely many $(-1)$-curves by Nagata [Nag60], and so the nef cone is not finite polyhedral. However, the automorphism group $\operatorname{Aut}(X)$ is trivial and, hence, does not have a finite polyhedral fundamental domain on the nef cone.

The conjecture also fails if we allow the $\mathbf{R}$-divisor $\Delta$ to have negative coefficients. Let $Y$ be a K3 surface whose cone of curves is not finite polyhedral, and let $X$ be the blow-up of $Y$ at a very general point. Then $(X,-E)$ is a klt Calabi-Yau pair, where $E$ is the exceptional curve. Here the cone of curves of $X$ is not finite polyhedral, but $\operatorname{Aut}(X)$ is trivial.

For $X$ terminal and $\Delta=0$, Conjecture 8.1 is exactly Kawamata's conjecture on Calabi-Yau fiber spaces, generalizing Morrison's conjecture on Calabi-Yau varieties [Kaw97, Mor93, Mor96]. (The group in part (2) can then be described as $\operatorname{Bir}(X / S)$, since all birational automorphisms of $X$ over $S$ are pseudo-automorphisms when $K_{X}$ is numerically trivial over $S$.) Assuming the base field has characteristic zero, Kawamata's conjecture is known for $X$ of dimension at most two, for $X$ of dimension three with $S$ of positive dimension, and for a few classes of Calabi-Yau three-folds [Kaw97, Remark 1.13]. Some evidence for Conjecture 8.1 in the case $\Delta \neq 0$ is provided by Coble surfaces, smooth projective rational surfaces such that the linear system $\left|-K_{X}\right|$ is empty but $\left|-2 K_{X}\right|$ is not empty. If there is a smooth divisor $D$ equivalent to $-2 K_{X}$, then $(X, D / 2)$ is a klt Calabi-Yau pair. Then Conjecture 8.1 is true for $(X, D / 2)$. Indeed, the double cover of $X$ ramified over $D$ is a $\mathrm{K} 3$ surface with at most ordinary double points [DZ01, Lemma 6.2], and the conjecture for $X$ follows from Oguiso and Sakurai [OS01, Corollary 1.9].

Conjecture 8.1 would have strong consequences for varieties with $-K_{X}$ semi-ample, as considered in this paper. Let $X$ be a $\mathbf{Q}$-factorial klt variety with $-K_{X}$ semi-ample. For any $m>1$ such that $-m K_{X}$ is basepoint-free, let $\Delta$ be $1 / m$ times a general divisor in the linear system $\left|-m K_{X}\right|$; then $(X, \Delta)$ is klt [KM98, Lemma 5.17]. Moreover, $K_{X}+\Delta \equiv 0$, and so $(X, \Delta)$ is a klt Calabi-Yau pair. Thus, Conjecture 8.1 implies that conclusions (1) and (2) hold whenever $-K_{X}$ is semi-ample. If $-K_{X}$ is ample, then the conjecture is known in characteristic zero: the nef cone is finite rational polyhedral 


\section{B. TOTARO}

by the cone theorem [KM98], and the movable cone is finite rational polyhedral, partitioned into the nef cones of the finitely many SQMs of $X$, by Birkar-Cascini-Hacon-McKernan [BCHM06].

We can now prove Conjecture 8.1 when $X$ is a rational elliptic surface; this completes the proof for all smooth projective surfaces with $-K_{X}$ semi-ample. For rational elliptic surfaces with no multiple fibers and Mordell-Weil rank eight (the maximum possible), Theorem 8.2 was already known, by Grassi and Morrison [GM93, Theorem 2.3].

Theorem 8.2. Let $X$ be a rational elliptic (or quasi-elliptic) surface over an algebraically closed field. That is, $X$ is a smooth projective surface with $-K_{X}$ semi-ample such that $T:=$ Proj $R\left(X,-K_{X}\right)$ has dimension one. Then the number of Aut $(X / T)$-equivalence classes of faces of the nef effective cone $A^{e}(X)$ corresponding to birational contractions or fiber space structures is finite. (In this case the nef effective cone is closed, thus equal to the nef cone.) Moreover, there exists a finite rational polyhedral cone $\Pi$ which is a fundamental domain for the action of $\operatorname{Aut}(X / T)$ on $A^{e}(X)$.

Also, the following are equivalent.

(1) The total coordinate ring of $X$ is finitely generated.

(2) The nef cone of $X$ is finite rational polyhedral.

(3) The Mordell-Weil group, $\mathrm{Pic}^{0}$ of the generic fiber of $X$ over T, is finite. (The Mordell-Weil group is automatically a finite-index subgroup of $\operatorname{Aut}(X / T)$.)

Note that we only have to consider statement (1) in Conjecture 8.1; statement (2) in the conjecture is vacuous for surfaces, because every movable divisor on a surface is nef. (Or, related to this: minimal models of surfaces are unique.)

Proof. The basic point is to show that the group $\operatorname{Aut}(X / T)$ has only finitely many orbits on the set of $(-1)$-curves in $X$.

The generic fiber of $X \rightarrow T$ is a curve $X_{\eta}$ of genus 1 over the function field of $T$. The class in $\operatorname{Pic}(X)$ of a general fiber of $X \rightarrow T$ is $-m K_{X}$ for some positive integer $m$. Here $-K_{X}$ is effective by Riemann-Roch, and so (if $m>1$ ) there is a multiple fiber of $X \rightarrow T$, a curve in the class of $-K_{X}$. (Here $m=1$ if and only if $X \rightarrow T$ has a section [CD89, ch. 5, §6].) The irreducible components of the reducible fibers of $X \rightarrow T$, if any, are exactly the (-2)-curves on $X$ (smooth curves $C$ of genus zero with $\left.K_{X} \cdot C=0\right)$. The Picard group $\operatorname{Pic}\left(X_{\eta}\right)$ is the quotient of $\operatorname{Pic}(X)$ by $-K_{X}$ together with all the (-2)-curves. The degree of a line bundle on $X$ on a general fiber of $X \rightarrow T$ is given by the intersection number with $-m K_{X}$, and so the Mordell-Weil group $G:=\operatorname{Pic}^{0}\left(X_{\eta}\right)$ is the subquotient of $\operatorname{Pic}(X)$ given by

$$
G=\left(K_{X}\right)^{\perp} /\left(-K_{X},(-2) \text {-curves }\right) .
$$

An element $x$ of the group $G$ acts by a translation on the curve $X_{\eta}$ of genus one and hence by an automorphism $\varphi_{x}$ on $X$, by Lemma 6.2. This gives an action of $G$ on $\operatorname{Pic}(X)$. We know how translation by an element $x$ of $\operatorname{Pic}^{0}\left(X_{\eta}\right)$ acts on $\operatorname{Pic}\left(X_{\eta}\right)$ : by $\varphi_{x}(y)=y+\operatorname{deg}(y) x$. Since $-m K_{X} \in \operatorname{Pic}(X)$ is the class of a general fiber of $X \rightarrow T$, this means that $\varphi_{x}$ acts on $\operatorname{Pic}(X)$ by $\varphi_{x}(y)=y-\left(m K_{X} \cdot y\right) x\left(\bmod -K_{X},(-2)\right.$-curves $)$. Using that the action of $G$ preserves the intersection product, we compute the action of certain elements of $G$ on $\operatorname{Pic}(X)$ by

$$
\varphi_{x}(y)=y-\left(m K_{X} \cdot y\right) x+\left[y \cdot\left(-x+(1 / 2)(x \cdot x) m K_{X}\right)\right]\left(-m K_{X}\right)
$$

for all $x \in K_{X}^{\perp}$ with $x \cdot C_{i}=0$ for all $(-2)$-curves $C_{i}$, and all $y \in \operatorname{Pic}(X)$.

Now let $E_{1}$ and $E_{2}$ be any two (-1)-curves on $X$ such that $E_{1} \cdot C_{i}=E_{2} \cdot C_{i}$ for all (-2)-curves $C_{i}$ and such that $E_{1} \equiv E_{2}(\bmod m \operatorname{Pic}(X))$. Let $x=\left(E_{2}-E_{1}\right) / m \in \operatorname{Pic}(X)$. Then $x$ is in $K_{X}^{\perp}$, we 


\section{HILBERT'S 14TH PROBLEM}

have $x \cdot C_{i}=0$ for all $(-2)$-curves $C_{i}$, and

$$
\varphi_{x}\left(E_{1}\right)=E_{1}-\left(m K_{X} \cdot E_{1}\right) x+\left[E_{1} \cdot\left(-m x+(1 / 2)(m x \cdot m x) K_{X}\right)\right]\left(-K_{X}\right)=E_{2} .
$$

Every $(-1)$-curve $E$ has $E \cdot\left(-m K_{X}\right)=m$, which says that $E$ is a multisection of degree $m$ of $X \rightarrow T$. Therefore, $0 \leqslant E \cdot C_{i} \leqslant m$ for each $(-2)$-curve $C_{i}$. So the $(-1)$-curves $E$ are divided into finitely many classes according to the intersection numbers of $E$ with all (-2)-curves and the class of $E$ in $\operatorname{Pic}(X) / m$. By the previous paragraph, the $(-1)$-curves on $X$ fall into finitely many orbits under the action of $G$. A fortiori, $\operatorname{Aut}(X / T)$ has finitely many orbits on the set of $(-1)$-curves.

We now describe all of the extremal rays of the cone of curves $\overline{N E}(X)$. By the cone theorem [KK94, Theorems 2.1.1 and 2.3.3], all of the $K_{X}$-negative extremal rays of $\overline{N E}(X)$ are spanned by $(-1)$-curves. Since $-K_{X}$ is nef, it remains to describe $\overline{N E}(X) \cap K_{X}^{\perp}$. It is a general fact, for any smooth projective surface $X$, that any extremal ray $\mathbf{R}^{>0} \cdot x$ of $\overline{N E}(X)$ with $x^{2}<0$ is spanned by a curve $C$ with $C^{2}<0$, and that such an extremal ray is isolated. Both statements follow from the fact that any two distinct curves $C$ and $D$ on $X$ with $C^{2}<0$ and $D^{2}<0$ have $C \cdot D \geqslant 0$ and hence are 'far' from each other.

For the rational elliptic surface $X$, the Hodge index theorem gives that the intersection pairing on $K_{X}^{\frac{1}{X}}$ is negative semidefinite, with $x^{2}=0$ only on the line spanned by $-K_{X}$. Therefore, using the fact in the previous paragraph, all extremal rays of $\overline{N E}(X) \cap K_{X}^{\perp}$ are spanned by either $-K_{X}$ or a $(-2)$-curve. There are only finitely many $(-2)$-curves (the irreducible components of reducible fibers of $X \rightarrow T$ ), and so the cone $\overline{N E}(X) \cap K_{X}^{\perp}$ is finite rational polyhedral. We conclude that every extremal ray of $\overline{N E}(X)$ is spanned by either a $(-1)$-curve or one of the finitely many (-2)-curves (or $-K_{X}$, if $X$ contains no (-2)-curve).

Since $\operatorname{Aut}(X / T)$ has only finitely many orbits on $(-1)$-curves, it follows that $\operatorname{Aut}(X / T)$ has only finitely many orbits on the extremal rays of $\overline{N E}(X)$. Moreover, if $X$ contains infinitely many $(-1)$-curves, then the only possible limit ray of $(-1)$-rays is $\mathbf{R}^{>0}\left(-K_{X}\right)$. Indeed, every $(-1)$-curve $E$ has $-K_{X} \cdot E=1$ and $E^{2}=-1$, while there are only finitely many $(-1)$-curves $E$ with any given degree $H \cdot E$ (a natural number), where $H$ is a fixed ample divisor on $X$. Any limit ray $\mathbf{R}^{>0} x$ of $(-1)$-rays has $0<H \cdot x<\infty$, and so it must have $\left(-K_{X}\right) \cdot x=0$ and $x^{2}=0$. Since the intersection form is negative semidefinite on $\left(K_{X}\right)^{\perp}$ with kernel spanned by $-K_{X}$, it follows that $x$ is a multiple of $-K_{X}$, as claimed.

We can deduce that the nef cone $\bar{A}(X)$ is finite rational polyhedral near any point $x$ in $\bar{A}(X)$ not in the ray $\mathbf{R}^{\geqslant 0}\left(-K_{X}\right)$. First, such a point $x$ has $x^{2} \geqslant 0$ and also $\left(-K_{X}\right) \cdot x \geqslant 0$, since $x$ and $-K_{X}$ are nef. If $\left(-K_{X}\right) \cdot x$ were equal to zero, these properties would imply that $x$ is a multiple of $-K_{X}$; hence we must have $\left(-K_{X}\right) \cdot x>0$. As a result, there is a neighborhood $V$ of $x$ and a neighborhood $W$ of $-K_{X}$ such that $V \cdot W>0$. Since the only possible limit ray of $(-1)$-rays is $\mathbf{R}^{>0}\left(-K_{X}\right)$, almost all (all but finitely many) (-1)-curves are in the cone $\mathbf{R}^{>0} W$. So almost all $(-1)$-curves have positive intersection with the neighborhood $V$ of $x$. Since almost all extremal rays of $\overline{N E}(X)$ are spanned by $(-1)$-curves, we conclude that the nef cone $\bar{A}(X)$ is finite rational polyhedral near $x$, as claimed.

In particular, for each $(-1)$-curve $E$, the face $\bar{A}(X) \cap E^{\perp}$ of the nef cone is finite rational polyhedral, since it does not contain $-K_{X}$. So the cone $\Pi_{E}$ spanned by $-K_{X}$ and $\bar{A}(X) \cap E^{\perp}$ is finite rational polyhedral.

Let $x$ be any nef $\mathbf{R}$-divisor on $X$. Let $c$ be the maximum real number such that $y:=x+c K_{X}$ is nef. Then $x$ and $y$ have the same degree on all $(-2)$-curves, and so there must be some $(-1)$-curve $E$ with $y \in E^{\perp}$. Therefore, $x$ is in the cone $\Pi_{E}$. That is, the nef cone $\bar{A}(X)$ is the union of the finite rational polyhedral cones $\Pi_{E}$. Moreover, for two distinct (-1)-curves $E$ and $F$ on $X$, the intersection Int $\Pi_{E} \cap \operatorname{Int} \Pi_{F}$ is empty, since $E$ and $F$ are linearly independent in $N^{1}(X)$. Since Aut $(X / T)$ has only finitely many orbits on the set of cones $\Pi_{E}$, there is a finite rational polyhedral cone $\Pi$ which 


\section{B. TOTARO}

is a fundamental domain for the action of $\operatorname{Aut}(X / T)$ on $A^{e}(X)$. (We can construct such a domain using the Dirichlet construction for the hyperbolic metric on $\left\{x \in N^{1}(X): x^{2}>0, H \cdot x>0\right\} / \mathbf{R}^{>0}$, restricted to the nef cone. That is, given a point $x$ in the interior of the nef cone, we define a fundamental domain as the set of points of the nef cone whose distance to $x$, in the nef cone modulo scalars, is at most their distance to any other point in the Aut $(X / T)$-orbit of $x$.)

Any rational point $x$ in the nef cone $\bar{A}(X)$ is effective. This is clear from the Riemann-Roch theorem $\chi(X, n L)=\chi(X, O)+\left((n L)^{2}-K_{X} \cdot n L\right) / 2$, since for a nef divisor $x$ on our surface $X$, either $x^{2}>0, x^{2}=0$ and $\left(-K_{X}\right) \cdot x>0$, or $x$ is a multiple of $-K_{X}$. Since the cone $\Pi_{E}$ is finite rational polyhedral for each $(-1)$-curve $E, \Pi_{E}$ is contained in the nef effective cone $A^{e}(X)$. Since the whole nef cone $\bar{A}(X)$ is the union of the cones $\Pi_{E}$, the nef effective cone $A^{e}(X)$ is equal to its closure, the nef cone $\bar{A}(X)$. This proves another statement of Theorem 8.2.

Next, we have to show that the number of $\operatorname{Aut}(X / T)$-orbits of faces of the nef effective cone $A^{e}(X)$ corresponding to birational contractions or fiber space structures is finite. Dually, it suffices to show that there are only finitely many $\operatorname{Aut}(X / T)$-orbits of faces in the cone of curves $\overline{N E}(X)$. Since there are only finitely many orbits of $(-1)$-curves, and almost all extremal rays of $\overline{N E}(X)$ are spanned by $(-1)$-curves, it suffices to show that each $(-1)$-curve $E$ lies on only finitely many faces of $\overline{N E}(X)$. This follows from the dual statement that the cone $\bar{A}(X) \cap E^{\perp}$ is finite rational polyhedral, which we have proved.

Finally, let us prove the equivalence of statements (1), (2) and (3) in Theorem 8.2. That statement (1) implies statement (2) is easy; it is also part of $\mathrm{Hu}$ and Keel's characterization of varieties with finitely generated total coordinate ring [HK00, Proposition 2.9]. Also, statement (2) easily implies statement (3), as follows. Suppose that the Mordell-Weil group $\operatorname{Pic}^{0}\left(X_{\eta}\right)$ is infinite. We know that $X$ contains at least one $(-1)$-curve since $X$ is a rational elliptic surface. The Mordell-Weil group $\operatorname{Pic}^{0}\left(X_{\eta}\right)$ acts on $X$, and only a finite subgroup of it can map any given (-1)-curve into itself, since a (-1)-curve is a multisection of $X \rightarrow T$ of degree a positive integer $m$. Thus, if $\mathrm{Pic}^{0}\left(X_{\eta}\right)$ is infinite, then $X$ has infinitely many $(-1)$-curves, and so $\overline{N E}(X)$ is not finite rational polyhedral. So the dual cone $\bar{A}(X)$ is not finite rational polyhedral. That is, statement (2) implies statement (3).

Conversely, suppose that statement (3) holds, that is, that $\operatorname{Pic}^{0}\left(X_{\eta}\right)$ is finite. This is a finiteindex subgroup of $\operatorname{Aut}(X / T)$, and so $\operatorname{Aut}(X / T)$ is finite. By the earlier parts of this theorem, it follows that the nef effective cone $A^{e}(X)$ is finite rational polyhedral. Statement (2) is proved. It remains to prove statement (1), that the total coordinate ring of $X$ is finitely generated. By $\mathrm{Hu}$-Keel's theorem [HK00, Proposition 2.9], it suffices to show that every codimension-one face of $\overline{N E}(X)$ can be contracted. This follows from the cone theorem (including the contraction theorem), by the same argument as in the proof of Theorem 5.2.

\section{ACKNOWLEDGEMENTS}

Thanks to Igor Dolgachev, Shigeru Mukai, and Greg Sankaran for their comments.

\section{Appendix A. Infinitely generated rings of invariants over $\mathbf{F}_{2}$ or $\mathbf{F}_{3}$}

In this appendix we complete the proof of Corollaries 5.1 and 7.1 by giving simple examples of 18-dimensional representations of $\left(G_{\mathrm{a}}\right)^{6}$ over $\mathbf{F}_{2}$ or $\mathbf{F}_{3}$, and 16-dimensional representations of $\left(G_{\mathrm{a}}\right)^{4}$ over $\mathbf{F}_{2}$, whose rings of invariants are not finitely generated.

Take the nine points in $\mathbf{P}^{2}\left(\mathbf{F}_{4}\right)$ given in affine coordinates by $(x, y)$ where $x$ and $y$ run through the nonzero elements of a field $\mathbf{F}_{4}$ of order four. These points are the intersection of the two cubics $x^{3}=z^{3}$ and $y^{3}=z^{3}$, each consisting of three lines through a point in $\mathbf{P}^{2}$. There are nine collinear triples among these points, and three partitions of them into disjoint collinear triples. 


\section{HILBERT'S 14TH PROBLEM}

(These partitions correspond to three cubics in the pencil which are unions of three lines, $x^{3}+z^{3}$, $y^{3}+z^{3}$, and $\left(x^{3}+z^{3}\right)+\left(y^{3}+z^{3}\right)=x^{3}+y^{3}$.) Therefore, $\rho=8-9+3=2$ is greater than zero. By Theorem 5.2, the blow-up $X$ of $\mathbf{P}^{2}$ at these nine points has infinitely many (-1)-curves. (More precisely, this count shows that the Mordell-Weil rank is two.) Thus, we get an 18-dimensional representation of $\left(G_{\mathrm{a}}\right)^{6}$ over $\mathbf{F}_{4}$ such that the ring of invariants is not finitely generated.

Since this pencil of cubics is defined over $\mathbf{F}_{2}$, even though the nine individual points are not, this pencil gives an 18-dimensional representation of $\left(G_{\mathrm{a}}\right)^{6}$ over $\mathbf{F}_{2}$ such that the ring of invariants is not finitely generated, as explained in $\S 4$. We write out this representation explicitly, although the reader might be satisfied to know that it can be done. Let $\zeta$ be a primitive cube root of unity in $\mathbf{F}_{4}$. Our representation of $\left(G_{\mathrm{a}}\right)^{6}$ on $A^{18}:=A_{1}^{9} \oplus A_{2}^{9}$ over $\mathbf{F}_{4}$ can be defined by six commuting linear maps $1+A_{i}, 1 \leqslant i \leqslant 6$, where $A_{i} \in \operatorname{Hom}\left(A_{2}^{9}, A_{1}^{9}\right)$ are given by the following diagonal matrices:

$$
\begin{aligned}
\operatorname{diag}\left(1, \zeta, \zeta^{2}, 0,0,0,0,0,0\right), & \operatorname{diag}\left(1,0,0, \zeta, \zeta^{2}, 0,0,0,0\right), \\
\operatorname{diag}\left(1,0,0,0,0, \zeta, \zeta^{2}, 0,0\right), & \operatorname{diag}(0,1,1,1,1,1,1,0,0), \\
\operatorname{diag}(0,0,0,0,0,1,1,1,1), & \operatorname{diag}\left(1,1,1,0,0,0,0, \zeta, \zeta^{2}\right) .
\end{aligned}
$$

These six vectors are chosen as a basis for the kernel of the linear map $A^{9} \rightarrow A^{3}$ corresponding to our nine points in $\mathbf{P}^{2}$ over $\mathbf{F}_{4}$, in the order $[1,1,1],[1, \zeta, 1],\left[1, \zeta^{2}, 1\right],[\zeta, 1,1],\left[\zeta^{2}, 1,1\right],[\zeta, \zeta, 1]$, $\left[\zeta^{2}, \zeta^{2}, 1\right],\left[\zeta, \zeta^{2}, 1\right],\left[\zeta^{2}, \zeta, 1\right]$. We can define a form of this representation over $\mathbf{F}_{2}$ by six commuting linear maps $1+A_{i}, 1 \leqslant i \leqslant 6$, where $A_{i} \in \operatorname{Hom}\left(A_{2}^{9}, A_{1}^{9}\right)$ are given by the following matrices:

$$
\begin{array}{cl}
\operatorname{diag}\left(1,\left(\begin{array}{ll}
0 & 1 \\
1 & 1
\end{array}\right), 0,0,0,0,0,0\right), & \operatorname{diag}\left(1,0,0,\left(\begin{array}{ll}
0 & 1 \\
1 & 1
\end{array}\right), 0,0,0,0\right) \\
\operatorname{diag}\left(1,0,0,0,0,\left(\begin{array}{ll}
0 & 1 \\
1 & 1
\end{array}\right), 0,0\right), & \operatorname{diag}(0,1,1,1,1,1,1,0,0) \\
\operatorname{diag}(0,0,0,0,0,1,1,1,1), & \operatorname{diag}\left(1,1,1,0,0,0,0,\left(\begin{array}{ll}
0 & 1 \\
1 & 1
\end{array}\right)\right) .
\end{array}
$$

This is an 18-dimensional representation of $\left(G_{\mathrm{a}}\right)^{6}$ over $\mathbf{F}_{2}$ with infinitely generated ring of invariants.

A similar example works over $\mathbf{F}_{3}$. Take the nine points of $\mathbf{P}^{2}\left(\mathbf{F}_{9}\right)$ given in affine coordinates by $(x, y)$ where $x$ and $y$ run through the set of fourth roots of unity in $\mathbf{F}_{9}$ other than one. These points are the intersection of the two cubics $x^{3}+x^{2} z+x z^{2}+z^{3}$ and $y^{3}+y^{2} z+y z^{2}+z^{3}$. There are seven lines through these points, and two partitions of them into disjoint collinear triples. (The two partitions correspond to two cubics in the pencil which are unions of three lines, $x^{3}+x^{2} z+x z^{2}+z^{3}$ and $y^{3}+y^{2} z+y z^{2}+z^{3}$, and the other collinear triple corresponds to another reducible cubic in the pencil, $\left(x^{3}+x^{2} z+x z^{2}+z^{3}\right)-\left(y^{3}+y^{2} z+y z^{2}+z^{3}\right)=(x-y)\left(x^{2}+x y+y^{2}+x z+y z+z^{2}\right)$.) So $\rho=8-7+2=3$. By Theorem 5.2, the blow-up $X$ of $\mathbf{P}^{2}$ at these nine points has infinitely many $(-1)$-curves. This gives an 18-dimensional representation of $\left(G_{\mathrm{a}}\right)^{6}$ over $\mathbf{F}_{9}$ whose ring of invariants is not finitely generated.

Since this pencil of cubics is defined over $\mathbf{F}_{3}$, we can define a form of the above representation over $\mathbf{F}_{3}$. Explicitly, let $i$ be a square root of -1 in $\mathbf{F}_{9}$. Then our representation of $\left(G_{\mathrm{a}}\right)^{6}$ on $A^{18}:=A_{1}^{9} \oplus A_{2}^{9}$ over $\mathbf{F}_{9}$ can be defined by six commuting linear maps $1+A_{j}, 1 \leqslant j \leqslant 6$, where $A_{j} \in \operatorname{Hom}\left(A_{2}^{9}, A_{1}^{9}\right)$ are given by the following diagonal matrices:

$$
\begin{aligned}
\operatorname{diag}(1,1+i, 1-i, 0,0,0,0,0,0), & \operatorname{diag}(1,0,0,1+i, 1-i, 0,0,0,0), \\
\operatorname{diag}(1,0,0,0,0,1+i, 1-i, 0,0), & \operatorname{diag}(1,1,1,1,1,-1,-1,0,0), \\
\operatorname{diag}(0,0,0,0,0,1,1,-1,-1), & \operatorname{diag}(0,-1,-1,1,1,-1,-1,1+i, 1-i) .
\end{aligned}
$$




\section{B. TOTARO}

These six vectors are chosen as a basis for the kernel of the linear map $A^{9} \rightarrow A^{3}$ corresponding to our nine points in $\mathbf{P}^{2}$ over $\mathbf{F}_{9}$, in the order $[-1,-1,1],[-1, i, 1],[-1,-i, 1],[i,-1,1],[-i,-1,1]$, $[i, i, 1],[-i,-i, 1],[i,-i, 1],[-i, i, 1]$. We can define a form of this representation over $\mathbf{F}_{3}$ by six commuting linear maps $1+A_{j}, 1 \leqslant j \leqslant 6$, where $A_{j} \in \operatorname{Hom}\left(A_{2}^{9}, A_{1}^{9}\right)$ are given by the following matrices:

$$
\begin{array}{ll}
\operatorname{diag}\left(1,\left(\begin{array}{rr}
0 & 1 \\
1 & -1
\end{array}\right), 0,0,0,0,0,0\right), & \operatorname{diag}\left(1,0,0,\left(\begin{array}{rr}
0 & 1 \\
1 & -1
\end{array}\right), 0,0,0,0\right), \\
\operatorname{diag}\left(1,0,0,0,0,\left(\begin{array}{rr}
0 & 1 \\
1 & -1
\end{array}\right), 0,0\right), & \operatorname{diag}(1,1,1,1,1,-1,-1,0,0), \\
\quad \operatorname{diag}(0,0,0,0,0,1,1,-1,-1), & \operatorname{diag}\left(0,-1,-1,1,1,-1,-1,\left(\begin{array}{rr}
0 & 1 \\
1 & -1
\end{array}\right)\right) .
\end{array}
$$

This is an 18-dimensional representation of $\left(G_{\mathrm{a}}\right)^{6}$ over $\mathbf{F}_{3}$ with infinitely generated ring of invariants. Corollary 5.1 is proved.

We now finish the proof of Corollary 7.1 by exhibiting a simple example of a 16-dimensional representation of $\left(G_{\mathrm{a}}\right)^{4}$ over $\mathbf{F}_{2}$ whose ring of invariants is not finitely generated.

Let $\zeta$ be a primitive cube root of unity in $\mathbf{F}_{4}$, and consider the eight points in $\mathbf{P}^{3}\left(\mathbf{F}_{4}\right)$ defined in affine coordinates $w=1$ by $(x, y, z)$ where $z$ is $\zeta$ or $\zeta^{2}, y$ is 0 or $z$, and $x$ is zero or one. These points are the complete intersection of the three quadrics $x^{2}+x w=0, y^{2}+y z=0$, and $z^{2}+z w+w^{2}=0$. Let $X$ be the blow-up of $\mathbf{P}^{3}$ at these eight points. We have an elliptic fibration $X \rightarrow \mathbf{P}^{2}$. We count that there are exactly 10 coplanar quadruples among the eight points, corresponding to five reducible quadrics in our net: $x(x+w), y(y+z), z^{2}+z w+w^{2}=(z+\zeta w)\left(z+\zeta^{2} w\right), y^{2}+y z+z^{2}+z w+w^{2}=$ $(y+w+\zeta z)\left(y+w+\zeta^{2} z\right)$, and $x^{2}+x w+z^{2}+z w+w^{2}=(x+z+\zeta w)\left(x+z+\zeta^{2} w\right)$. Since $\rho=7-\frac{1}{2}(10)=2$ is greater than zero, Theorem 7.2 shows that the Mordell-Weil group of the general fiber $E$ of $X$ over $k\left(\mathbf{P}^{2}\right)$ is infinite (in fact, of rank two), and therefore the ring of invariants for the given 16-dimensional representation of $\left(G_{\mathrm{a}}\right)^{4}$ over $\mathbf{F}_{4}$ is not finitely generated.

Since this net of quadrics is defined over $\mathbf{F}_{2}$, even though the eight individual points are not, we can define a form of the above representation over $\mathbf{F}_{2}$. Our representation of $\left(G_{\mathrm{a}}\right)^{4}$ on $A^{16}:=A_{1}^{8} \oplus A_{2}^{8}$ over $\mathbf{F}_{4}$ can be defined by 4 commuting linear maps $1+A_{i}, 1 \leqslant i \leqslant 4$, where $A_{i} \in \operatorname{Hom}\left(A_{2}^{8}, A_{1}^{8}\right)$ are given by the following diagonal matrices:

$$
\begin{aligned}
\operatorname{diag}(0,0,1,1,0,0,1,1), & \operatorname{diag}\left(0,0,0,0, \zeta, \zeta^{2}, \zeta, \zeta^{2}\right), \\
\operatorname{diag}\left(\zeta, \zeta^{2}, \zeta, \zeta^{2}, \zeta, \zeta^{2}, \zeta, \zeta^{2}\right), & \operatorname{diag}(1,1,1,1,1,1,1,1) .
\end{aligned}
$$

These four vectors are chosen as a basis for the kernel of the linear map $A^{8} \rightarrow A^{4}$ corresponding to our eight points in $\mathbf{P}^{2}$ over $\mathbf{F}_{4}$, in the order $[0,0, \zeta, 1],\left[0,0, \zeta^{2}, 1\right],[1,0, \zeta, 1],\left[1,0, \zeta^{2}, 1\right],[0, \zeta, \zeta, 1]$, $\left[0, \zeta^{2}, \zeta^{2}, 1\right],[1, \zeta, \zeta, 1],\left[1, \zeta^{2}, \zeta^{2}, 1\right]$. We can define a form of this representation over $\mathbf{F}_{2}$ by four commuting linear maps $1+A_{i}, 1 \leqslant i \leqslant 4$, where $A_{i} \in \operatorname{Hom}\left(A_{2}^{8}, A_{1}^{8}\right)$ are given by the following matrices:

$$
\begin{gathered}
\operatorname{diag}(0,0,1,1,0,0,1,1), \quad \operatorname{diag}\left(0,0,0,0,\left(\begin{array}{ll}
0 & 1 \\
1 & 1
\end{array}\right),\left(\begin{array}{ll}
0 & 1 \\
1 & 1
\end{array}\right)\right), \\
\operatorname{diag}\left(\left(\begin{array}{ll}
0 & 1 \\
1 & 1
\end{array}\right),\left(\begin{array}{ll}
0 & 1 \\
1 & 1
\end{array}\right),\left(\begin{array}{ll}
0 & 1 \\
1 & 1
\end{array}\right),\left(\begin{array}{ll}
0 & 1 \\
1 & 1
\end{array}\right)\right), \quad \operatorname{diag}(1,1,1,1,1,1,1,1) .
\end{gathered}
$$

This is a 16-dimensional representation of $\left(G_{\mathrm{a}}\right)^{4}$ over $\mathbf{F}_{2}$ with infinitely generated ring of invariants. That completes the proof of Corollary 7.1. 


\section{HILBERT'S 14TH PROBLEM}

\section{REFERENCES}

Bea96 A. Beauville, Complex algebraic surfaces (Cambridge University Press, Cambridge, 1996).

BCHM06 C. Birkar, P. Cascini, C. Hacon and J. McKernan, Existence of minimal models for varieties of log general type, Preprint (2006), math.AG/0610203.

Cob29 A. Coble, Algebraic geometry and theta functions (American Mathematical Society, Providence, RI, 1929).

CD89 F. Cossec and I. Dolgachev, Enriques surfaces I (Birkhäuser, Basel, 1989).

Dol04 I. Dolgachev, On certain families of elliptic curves in projective space, Ann. Mat. Pura Appl. (4) 183 (2004), 317-331.

DO88 I. Dolgachev and D. Ortland, Point sets in projective spaces and theta functions, Astérisque, vol. 165 (Société Mathématiq́ue de France, Paris, 1988).

DZ01 I. Dolgachev and D.-Q. Zhang, Coble rational surfaces, Amer. J. Math. 123 (2001), 79-114.

Fre07 G. Freudenburg, A linear counterexample to Hilbert's fourteenth problem in dimension eleven, Proc. Amer. Math. Soc. 135 (2007), 51-57.

GM93 A. Grassi and D. Morrison, Automorphisms and the Kähler cone of certain Calabi-Yau manifolds, Duke Math. J. 71 (1993), 831-838.

HK00 Y. Hu and S. Keel, Mori dream spaces and GIT, Michigan Math. J. 48 (2000), 331-348.

Kaw97 Y. Kawamata, On the cone of divisors of Calabi-Yau fiber spaces, Internat. J. Math. 8 (1997), 665-687.

KK94 J. Kollár and S. Kovács, Birational geometry of log surfaces, 1994, http://www.math.princeton. edu/ ${ }^{\sim}$ kollar

KM98 J. Kollár and S. Mori, Birational geometry of algebraic varieties (Cambridge University Press, Cambridge, 1998).

Kur04 S. Kuroda, A generalization of Roberts' counterexample to the fourteenth problem of Hilbert, Tohoku Math. J. 56 (2004), 501-522.

Lan91 W. Lang, Extremal rational elliptic surfaces in characteristic p. I. Beauville surfaces, Math. Z. 207 (1991), 429-437.

Lan94 W. Lang, Extremal rational elliptic surfaces in characteristic p. II. Surfaces with three or fewer singular fibres, Ark. Mat. 32 (1994), 423-448.

Man64 Y. Manin, The Tate height of points on an abelian variety: its variants and applications, Izv. Akad. Sci. SSSR 28 (1964), 1363-1390; AMS Transl. 59 (1966), 82-119.

MP86 R. Miranda and U. Persson, On extremal rational elliptic surfaces, Math. Z. 193 (1986), 537-558.

Mor93 D. Morrison, Compactifications of moduli spaces inspired by mirror symmetry, in Journées de géométrie algébrique d'Orsay (Orsay, 1992), Astérisque, vol. 218 (Société Mathématique de France, Paris, 1993), 243-271.

Mor96 D. Morrison, Beyond the Kähler cone, in Proceedings of the Hirzebruch 65 conference on algebraic geometry (Ramat Gan, 1993) (Bar-Ilan University, 1996), 361-376.

Muk01 S. Mukai, Counterexample to Hilbert's fourteenth problem for the 3-dimensional additive group, RIMS Preprint 1343 (2001).

Nag58 M. Nagata, On the fourteenth problem of Hilbert, in Proc. Int. Conf. Mathematics (Edinburgh, 1958), 459-462.

Nag60 M. Nagata, On rational surfaces. II, Mem. Coll. Sci. Univ. Kyoto Ser. A Math. 33 (1960/1961), 271-293.

Nik00 V. Nikulin, A remark on algebraic surfaces with polyhedral Mori cone, Nagoya Math. J. 157 (2000), 73-92.

Nik04 V. Nikulin, On algebraic varieties with finite polyhedral Mori cone in Proc. the Fano Conference (University of Torino, 2004), 573-589.

OS01 K. Oguiso and J. Sakurai, Calabi-Yau threefolds of quotient type, Asian. J. Math. 5 (2001), 43-77. 


\section{HILBERT'S 14TH PROBLEM}

Pre08 A. Prendergast-Smith, Finite generation of the total coordinate ring for certain rational threefolds, Preprint (2008).

Ser94 J.-P. Serre, Cohomologie galoisienne, Lecture Notes in Mathematics, vol. 5, 5th edition (Springer, Berlin, 1994).

Shi72 T. Shioda, On elliptic modular surfaces, J. Math. Soc. Japan 24 (1972), 20-59.

Ste97 R. Steinberg, Nagata's example, in Algebraic groups and Lie groups (Cambridge University Press, Cambridge, 1997), 375-384.

Tat65 J. Tate, On the conjecture of Birch and Swinnerton-Dyer, in Séminaire Bourbaki, Vol. 9, 1964/651965/66, Exposé 306 (Société Mathematique de France, Paris 1995), 415-440.

Burt Totaro b.totaro@dpmms.cam.ac.uk

DPMMS, Wilberforce Road, Cambridge CB3 0WB, UK 